\title{
Pacific
}

Journal of

Mathematics

\section{MIXED INTERIOR AND BOUNDARY NODAL BUBBLING SOLUTIONS FOR A sinh-POISSON EQUATION}

JUNCHENG WeI, LONG WeI AND FENG ZhOU 


\title{
MIXED INTERIOR AND BOUNDARY NODAL BUBBLING SOLUTIONS FOR A sinh-POISSON EQUATION
}

\author{
Juncheng Wei, Long Wei AND Feng Zhou
}

We consider here the semilinear equation $\Delta u+2 \varepsilon^{2} \sinh u=0$ posed on a bounded smooth domain $\Omega$ in $\mathbb{R}^{2}$ with homogeneous Neumann boundary condition, where $\varepsilon>0$ is a small parameter. We show that for any given nonnegative integers $k$ and $l$ with $k+l \geq 1$, there exists a family of solutions $u_{\varepsilon}$ that develops $2 k$ interior and $2 l$ boundary singularities for $\varepsilon$ sufficiently small, with the property that

$$
2 \varepsilon^{2} \sinh u_{\varepsilon}-8 \pi \sum_{i=1}^{2 k}(-1)^{i-1} \delta_{\xi_{i}}+4 \pi \sum_{i=1}^{2 l}(-1)^{i-1} \delta_{\xi_{i}},
$$

where $\left(\xi_{1}, \ldots, \xi_{2(k+l)}\right)$ are critical points of some functional defined explicitly in terms of the associated Green function.

\section{Introduction}

The two-dimensional sinh-Poisson equation

$$
\Delta u+2 \varepsilon^{2} \sinh u=0
$$

arises in various important contexts, notably as a vorticity equation in classical hydrodynamics [Gurarie and Chow 2004; Chow et al. 1998; Kuvshinov and Schep 2000; Mallier and Maslowe 1993], in physico-chemical hydrodynamics [Probstein 1994] and in the geometry of constant mean curvature surfaces [Wente 1986]. In the vorticity connection, it occurs in a remarkable manner out of natural relaxation states in the long-time computation of two-dimensional fluid motion [Mallier and Maslowe 1993] (see also the references therein). In geometry, the sinh-Poisson equation plays a very important role in the study of the construction of constant mean curvature surfaces initiated by Wente [1986]. Wente's seminal work then

J. Wei is supported by an earmarked grant CUHK4238/01P from RGC, Hong Kong. L. Wei is partly supported by NSFC 10926057 and Foundation of Zhejiang Educational Committee under grant number Y200908784. F. Zhou is supported in part by NSFC number 10971067 and Shanghai project 09XD1401600.

MSC2000: 35J20, 35J65.

Keywords: boundary-interior nodal bubbling solutions, sinh-Poisson equation. 
led to work by Steffen [1986], Struwe [1986] and Brezis and Coron [1984], which completed the understanding of the blow-up for constant mean curvature surfaces from a geometric point of view. Spruck [1988] was the first to study the sinhPoisson equation from an analytic point of view. Recently, the asymptotic behavior of solutions to (1-1) was studied on a closed Riemann surface in [Ohtsuka and Suzuki 2006] and [Jost et al. 2008]. The authors applied the so-called "symmetrization method" and "Pohozaev identity", respectively, to show that there possibly exist two different types of blow-up for a family of solutions to (1-1). Conversely, Bertolucci and Pistoia [2007] tried to construct blow-up solutions to (1-1) with Dirichlet boundary conditions for $n=2$, and proved that for $\varepsilon$ positive and small enough, there exist at least two pairs of solutions that change sign exactly once, that concentrate in the domain and that have their nodal lines intersecting the boundary.

In [Wei et al. 2011] and [Wei 2009] the Neumann problem

$$
\left\{\begin{aligned}
\Delta u+2 \varepsilon^{2} \sinh u=0 & & \text { in } \Omega, \\
\partial u / \partial v=0 & & \text { on } \partial \Omega
\end{aligned}\right.
$$

was considered, where $\Omega$ is a bounded domain in $\mathbb{R}^{2}$ with smooth boundary $\partial \Omega$ and $\varepsilon>0$ is a parameter. The authors showed a concentration phenomena of solutions to (1-2) in the domain in [Wei et al. 2011], and on the boundary in [Wei 2009].

In this paper, we continue the study of the existence of solutions to (1-2). We prove that there exists a family of solutions $u_{\varepsilon}$ that concentrate positively and negatively in the domain and its boundary.

To state our results, we need to introduce some notation. First, let us define the corresponding Green function for the Neumann problem:

$$
\left\{\begin{aligned}
-\Delta G(x, y) & =\delta_{y}(x)-1 /|\Omega| & & \text { in } \Omega, \\
\partial G / \partial \nu & =0 & & \text { on } \partial \Omega, \\
\int_{\Omega} G(x, y) d x & =0 . & &
\end{aligned}\right.
$$

The regular part of $G(x, y)$ is defined depending on whether $y$ lies in the domain or on its boundary as

$$
H(x, y)= \begin{cases}G(x, y)+\frac{1}{2 \pi} \log |x-y| & \text { for } y \in \Omega, \\ G(x, y)+\frac{1}{\pi} \log |x-y| & \text { for } y \in \partial \Omega .\end{cases}
$$

In this way, $H(\cdot, y)$ is of class $C^{1, \alpha}$ in $\bar{\Omega}$.

For $k+l \geq 1$ and points $\xi_{j}$ for $j=1, \ldots, 2(k+l)$, with $\xi_{j} \in \Omega$ for $j \leq 2 k$ and $\xi_{j} \in \partial \Omega$ for $2 k+1 \leq j \leq 2(k+l)$, we define

$$
\varphi_{2(k+l)}\left(\xi_{1}, \ldots, \xi_{2(k+l)}\right)=\sum_{j=1}^{2(k+l)} c_{j}^{2} H\left(\xi_{j}, \xi_{j}\right)+\sum_{j \neq i} c_{j} c_{i}(-1)^{j+i} G\left(\xi_{j}, \xi_{i}\right)
$$


and denote

$$
\begin{aligned}
\mu_{d}:=\left\{\xi=\left(\xi_{1}, \ldots, \xi_{2 k}, \xi_{2 k+1}, \ldots,\right.\right. & \left.\xi_{2(k+l)}\right) \in \Omega^{2 k} \times \partial \Omega^{2 l} \\
& \left.\left|\min _{j \neq i}\right| \xi_{j}-\xi_{i} \mid \geq d, \min _{j=1, \ldots, 2 k} \operatorname{dist}\left(\xi_{j}, \partial \Omega\right) \geq d\right\},
\end{aligned}
$$

where $c_{i}=8 \pi$ for $i=1, \ldots, 2 k$ and $c_{i}=4 \pi$ for $i=2 k+1, \ldots, 2(k+l)$.

Definition 1.1 [Esposito et al. 2006]. We say that $\xi$ is a $C^{0}$-stable critical point of $\varphi_{m}: \mu_{d} \rightarrow \mathbb{R}$ if for any sequence of functions $\varphi_{m}^{n}: \mu_{d} \rightarrow \mathbb{R}$ such that $\varphi_{m}^{n} \rightarrow \varphi_{m}$ uniformly on compact sets of $\mathcal{M}_{d}$, the function $\varphi_{m}^{n}$ has a critical point $\xi_{n}$ such that $\varphi_{m}^{n}\left(\xi_{n}\right) \rightarrow \varphi_{m}(\xi)$.

In particular, if $\xi$ is a strict local minimum/maximum point of $\varphi_{m}$, then $\xi$ is a $C^{0}$-stable critical point.

Theorem 1.2 (main result). Let $k$ and $l$ be nonnegative integers with $k+l \geq 1$. Assume $\xi^{*} \in \mathcal{M}_{d}$ is a $C^{0}$-stable critical point of $\varphi_{2(k+l)}$. Then for any sufficiently small $\varepsilon>0$, there is a solution $u_{\varepsilon}$ to (1-2) with the property that

$$
2 \varepsilon^{2} \int_{\Omega}\left|\sinh u_{\varepsilon}\right| d x \rightarrow 8 \pi(2 k+l) \quad \text { as } \varepsilon \rightarrow 0 .
$$

More precisely, for any sequence $\left\{\varepsilon_{n}\right\}_{n \geq 1}$ that tends to 0 , there is a subsequence and $2(k+l)$ points $\xi_{i} \in \bar{\Omega}$ for $i=1, \ldots, 2(k+l)$, with $\xi_{j} \in \Omega$ for $j \leq 2 k$ and $\xi_{j} \in \partial \Omega$ for $2 k+1 \leq j \leq 2(k+l)$, and positive constants $\mu_{i}$ for $i=1, \ldots, 2(k+l)$ such that

$$
u_{\varepsilon}(x)=\sum_{i=1}^{2(k+l)}(-1)^{i-1}\left(\log \frac{1}{\left(\varepsilon^{2} \mu_{i}^{2}+\left|x-\xi_{i}\right|^{2}\right)^{2}}+c_{i} H\left(x, \xi_{i}\right)\right)+o(1)
$$

and

$$
2 \varepsilon^{2} \sinh u_{\varepsilon} \rightarrow 8 \pi \sum_{i=1}^{2 k}(-1)^{i-1} \delta_{\xi_{i}}+4 \pi \sum_{i=2 k+1}^{2(k+l)}(-1)^{i-1} \delta_{\xi_{i}}
$$

in the sense of measure. Moreover, the constants $\mu_{i}$ are given by

$$
\log \left(8 \mu_{i}^{2}\right)=c_{i} H\left(\xi_{i}, \xi_{i}\right)+\sum_{j \neq i}(-1)^{j+i} c_{j} G\left(\xi_{i}, \xi_{j}\right) .
$$

The $l=0$ (or $k=0$ ) case of this theorem was proved in [Wei et al. 2011] (or [Wei 2009]). The conditions that $\xi^{*} \in M_{d}$ be a $C^{0}$-stable critical point of $\varphi_{2(k+l)}$ is perhaps not necessary. Here, we need it only because of the technique we will use. In particular, for the case $k=l=1$ and $\Omega=B=B(0,1)$, the unit ball in $\mathbb{R}^{2}$, we don't need the condition and can obtain the existence and the profile of sign-changing solutions that concentrate positively and negatively at different points $\xi_{1}, \xi_{2} \in B$ and $\xi_{3}, \xi_{4} \in \partial B$. More precisely: 
Theorem 1.3. Let $k=l=1$. Then, there exists a solution $u_{\varepsilon}$ to (1-2) that concentrates at different points $\xi_{1}, \xi_{2} \in B$ and $\xi_{3}, \xi_{4} \in \partial B$, according to (1-6), (1-7) and (1-8) with $k=l=1$, as $\varepsilon$ goes to 0 .

Del Pino and Wei [2006] considered the problem $-\Delta u+u=\lambda e^{u}$ under Neumann boundary conditions and built a solution with $\lambda \int_{\Omega} e^{u}$ uniformly bounded and boundary-interior concentrating, such that $\lambda e^{u} \rightarrow 8 \pi \sum_{j=1}^{k} \delta_{\xi_{j}}+4 \pi \sum_{j=k+1}^{m} \delta_{\xi_{j}}$. For basic cells, they used explicit solutions of

$$
\Delta u+e^{u}=0 \quad \text { in } \mathbb{R}^{2}, \quad \int_{\mathbb{R}^{2}} e^{u} d x<+\infty
$$

given by

$$
U_{\mu, \xi}=\log \frac{8 \mu^{2}}{\left(\mu^{2}+|x-\xi|^{2}\right)^{2}} \quad \text { for } \mu>0 \text { and } \xi \in \mathbb{R}^{2} .
$$

In this paper, we will also construct solutions predicted by the theorems using these ones, but suitably scaled and projected so that it works for the nonlinearity we consider here. A special feature of our problem is presence of mixed positive-negative boundary-interior bubbling solutions. This is a new concentration phenomenon. To capture such solutions, we use the so-called localized energy method, which combines Lyapunov-Schmidt reduction and variational techniques. Such a scheme was been used in many works; see for instance [Dávila et al. 2005; del Pino et al. 2005; del Pino and Wei 2006] and references therein. Here we follow [del Pino and Wei 2006; Wei et al. 2011; Wei 2009], but we will overcome some of the difficulties that the mixed concentration phenomenon brings by delicate analysis.

\section{Ansatz for the solution}

In this section we will provide a first approximation for the solution of the problem (1-2) predicted by Theorems 1.2 and 1.3. Let us fix $k+l \geq 1$. For $i=1, \ldots, 2(k+l)$, let $\xi_{i} \in \bar{\Omega}$ and let $\mu_{i}$ be positive numbers to be chosen later. We define

$$
u_{i}(x)=\log \frac{8 \mu_{i}^{2}}{\left(\varepsilon^{2} \mu_{i}^{2}+\left|x-\xi_{i}\right|^{2}\right)^{2}} .
$$

The ansatz is

$$
U(x)=\sum_{i=1}^{2(k+l)}(-1)^{i-1}\left(u_{i}(x)+H_{i}^{\varepsilon}(x)\right)
$$

where $H_{i}^{\varepsilon}(x)$ is a correction term defined as the solution of

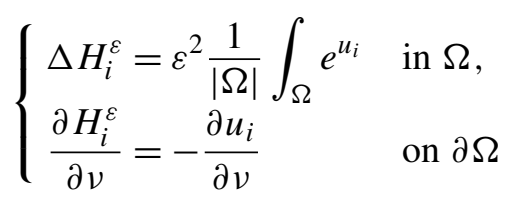


with the property that

$$
\int_{\Omega} H_{i}^{\varepsilon}(x) d x=-\int_{\Omega} u_{i} d x .
$$

This function resembles the shape of the regular part of the Green's function. Indeed, the following estimate for $H_{i}^{\varepsilon}$ holds true.

Lemma 2.1. For any $0<\alpha<1$

$$
H_{i}^{\varepsilon}(x)=c_{i} H\left(x, \xi_{i}\right)-\log \left(8 \mu_{i}^{2}\right)+O(\varepsilon)
$$

holds uniformly in $\bar{\Omega}$, where $H$ is the regular part of the Green function defined by (1-4).

Proof. The regular part of Green's function $H\left(x, \xi_{i}\right)$ satisfies

$$
\begin{cases}\Delta H\left(x, \xi_{i}\right)=\frac{1}{|\Omega|} & \text { in } \Omega, \\ \frac{\partial H}{\partial v}\left(x, \xi_{i}\right)=\frac{4}{c_{i}} \frac{\left(x-\xi_{i}\right) \cdot v(x)}{\left|x-\xi_{i}\right|^{2}} & \text { on } \partial \Omega .\end{cases}
$$

Now we define $z_{\varepsilon}(x)=H_{i}^{\varepsilon}(x)+\log \left(8 \mu_{i}^{2}\right)-c_{i} H\left(x, \xi_{i}\right)$. Then

$$
\begin{cases}\Delta z_{\varepsilon}=\varepsilon^{2} \frac{1}{|\Omega|} \int_{\Omega} e^{u_{i}}-\frac{c_{i}}{|\Omega|} & \text { in } \Omega, \\ \frac{\partial z_{\varepsilon}}{\partial v}=4 \frac{\left(x-\xi_{i}\right) \cdot v(x)}{\varepsilon^{2} \mu_{i}^{2}+\left|x-\xi_{i}\right|^{2}}-4 \frac{\left(x-\xi_{i}\right) \cdot v(x)}{\left|x-\xi_{i}\right|^{2}} & \text { on } \partial \Omega .\end{cases}
$$

First, by the definition of $u_{i}$, we have

$$
\begin{aligned}
\varepsilon^{2} \int_{\Omega} e^{u_{i}} & =\varepsilon^{2} \int_{\Omega} \frac{8 \mu_{i}^{2}}{\left(\varepsilon^{2} \mu_{i}^{2}+\left|x-\xi_{i}\right|^{2}\right)^{2}} \\
& =8 \varepsilon^{2} \int_{\Omega / \varepsilon \mu_{i}} \frac{\mu_{i}^{2}}{\left(\varepsilon^{2} \mu_{i}^{2}+\varepsilon^{2} \mu_{i}^{2} y^{2}\right)^{2}} \varepsilon^{2} \mu_{i}^{2} \\
& =8 \int_{\Omega / \varepsilon \mu_{i}} \frac{d y}{\left(1+y^{2}\right)^{2}} \\
& =2 c_{i}\left(\int_{0}^{\infty} \frac{t d t}{\left(1+t^{2}\right)^{2}}+O\left(\int_{1 / \varepsilon \mu_{i}}^{\infty} \frac{t d t}{\left(1+t^{2}\right)^{2}}\right)\right) \\
& =c_{i}+O\left(\varepsilon^{2} \mu_{i}^{2}\right)
\end{aligned}
$$

Next, for $\xi_{i} \in \Omega$ with $i=1, \ldots, 2 k$, we have

$$
\frac{\partial H_{i}^{\varepsilon}}{\partial v}=4 \frac{\left(x-\xi_{i}\right) \cdot v(x)}{\varepsilon^{2} \mu_{i}^{2}+\left|x-\xi_{i}\right|^{2}}=4 \frac{\left(x-\xi_{i}\right) \cdot v(x)}{\left|x-\xi_{i}\right|^{2}}+O\left(\varepsilon^{2}\right) \quad \text { for all } \xi_{i} \in \Omega, x \in \partial \Omega .
$$


For $\xi_{i} \in \partial \Omega$ with $i=2 k+1, \ldots, 2(k+l)$, we have

$$
\lim _{\varepsilon \rightarrow 0} \frac{\partial H_{i}^{\varepsilon}}{\partial v}=4 \frac{\left(x-\xi_{i}\right) \cdot v(x)}{\left|x-\xi_{i}\right|^{2}} \text { for all } x \neq \xi_{i} .
$$

We claim that for any $p>1$ there exists $C>0$ such that

$$
\left\|\frac{\partial H_{i}^{\varepsilon}}{\partial v}-4 \frac{\left(x-\xi_{i}\right) \cdot v(x)}{\left|x-\xi_{i}\right|^{2}}\right\|_{L^{p}(\partial \Omega)} \leq C \varepsilon^{1 / p} .
$$

It is not difficult to prove that the inequality

$$
\left|\left(x-\xi_{i}\right) \cdot v(x)\right| \leq C\left|x-\xi_{i}\right|^{2} \quad \text { for all } x \in \partial \Omega
$$

holds for $\xi_{i} \in \partial \Omega$ by assuming that $\xi_{i}=0$ and that near the origin $\partial \Omega$ is the graph of a function $P:(-\delta, \delta) \rightarrow \mathbb{R}$ with $P(0)=P^{\prime}(0)=0$. Now from (2-10) we obtain

$$
\begin{aligned}
\left|\frac{\partial H_{i}^{\varepsilon}}{\partial v}-4 \frac{\left(x-\xi_{i}\right) \cdot v(x)}{\left|x-\xi_{i}\right|^{2}}\right| & =4 \varepsilon^{2} \mu_{i}^{2} \frac{\left|\left(x-\xi_{i}\right) \cdot v(x)\right|}{\left|x-\xi_{i}\right|^{2}\left(\varepsilon^{2} \mu_{i}^{2}+\left|x-\xi_{i}\right|^{2}\right)} \\
& \leq \frac{C \varepsilon^{2}}{\varepsilon^{2} \mu_{i}^{2}+\left|x-\xi_{i}\right|^{2}} .
\end{aligned}
$$

Thus for $\lambda>0$ small but fixed,

$$
\left|\frac{\partial H_{i}^{\varepsilon}}{\partial v}-4 \frac{\left(x-\xi_{i}\right) \cdot v(x)}{\left|x-\xi_{i}\right|^{2}}\right| \leq C \varepsilon^{2} \quad \text { for all }\left|x-\xi_{i}\right| \geq \lambda, x \in \partial \Omega .
$$

Letting $p>1$ and changing variables $x-\xi_{i}=\varepsilon y \mu_{i}$, we have

$$
\begin{aligned}
\int_{B_{\lambda}\left(\xi_{i}\right) \cap \partial \Omega}\left|\frac{\varepsilon^{2}}{\varepsilon^{2} \mu_{i}^{2}+\left|x-\xi_{i}\right|^{2}}\right|^{p} & =C \varepsilon \int_{B_{\lambda / \varepsilon \mu_{i}}(0) \cap \partial \Omega_{\varepsilon}}\left|\frac{1}{1+|y|^{2}}\right|^{p} d y \\
& =C \varepsilon \int_{0}^{\lambda / \varepsilon \mu_{i}} \frac{1}{\left(1+t^{2}\right)^{p}} d t \leq C \varepsilon .
\end{aligned}
$$

This, combined with (2-11) and (2-12), shows that (2-9) holds.

By elliptic regularity theory, we obtain $z_{\varepsilon} \in W^{1+s, p}(\Omega)$ for any $p \geq 1$, with $0<s<1 / p$. On the other hand, from the Poincaré inequality we get

$$
\left\|z_{\varepsilon}-\frac{1}{|\Omega|} \int_{\Omega} z_{\varepsilon}\right\|_{W^{1+s, p}(\Omega)} \leq C\left\|\nabla z_{\varepsilon}\right\|_{L^{p}(\Omega)} \leq C \varepsilon^{1 / p} .
$$

This implies the existence of a constant $M$ such that

$$
z_{\varepsilon}(x)=M+O\left(\varepsilon^{\alpha}\right) \quad \text { for any } \alpha \in(0,1),
$$

uniformly in $\bar{\Omega}$, where $M=\lim _{\varepsilon \rightarrow 0}|\Omega|^{-1} \int_{\Omega} z_{\varepsilon} d x$. 
To obtain the result, we only need to show $M=0$. First, by the definition of $z_{\varepsilon}$ we have

$$
M=\lim _{\varepsilon \rightarrow 0}\left(\frac{1}{|\Omega|} \int_{\Omega} H_{i}^{\varepsilon}(x) d x+\log \left(8 \mu_{i}^{2}\right)-\frac{c_{i}}{|\Omega|} \int_{\Omega} H\left(x, \xi_{i}\right) d x\right) .
$$

The direct computation from (2-4) shows that

$$
\begin{aligned}
\int_{\Omega} H_{i}^{\varepsilon}(x) & =-\int_{\Omega}\left(\log \left(8 \mu_{i}^{2}\right)+\log \frac{1}{\left(\varepsilon^{2} \mu_{i}^{2}+\left|x-\xi_{i}\right|^{2}\right)^{2}}\right) \\
& =-|\Omega| \log \left(8 \mu_{i}^{2}\right)+2 \int_{\Omega} \log \left(1+\frac{\varepsilon^{2} \mu_{i}^{2}}{\left|x-\xi_{i}\right|^{2}}\right)-4 \int_{\Omega} \log \frac{1}{\left|x-\xi_{i}\right|} \\
& =-|\Omega| \log \left(8 \mu_{i}^{2}\right)+c_{i} \int_{\Omega} H\left(x, \xi_{i}\right) d x+O\left(\varepsilon^{2} \log \varepsilon^{-1}\right),
\end{aligned}
$$

where the last equality is consequence of the definition of $H$ and the property of the Green function. Therefore (2-13) implies $M=0$.

In $\Omega_{\varepsilon}=\Omega / \varepsilon$, let $v(y)=u(\varepsilon y)$; then solving problem (1-2) is equivalent to solving

$$
\left\{\begin{aligned}
\Delta v(y)+2 \varepsilon^{4} \sinh v=0 & \text { in } \Omega_{\varepsilon}, \\
\partial v / \partial v=0 & \text { on } \partial \Omega_{\varepsilon} .
\end{aligned}\right.
$$

We will seek a solution $v$ of (2-14) of the form

$$
v(y)=V(y)+\phi(y) \quad \text { for all } y \in \Omega_{\varepsilon},
$$

where

$$
V(y)=\sum_{i=1}^{2(k+l)}(-1)^{i-1}\left(u_{i}(\varepsilon y)+H_{i}^{\varepsilon}(\varepsilon y)\right) .
$$

Problem (2-14) can be restated: Find a solution $\phi$ to

$$
\left\{\begin{aligned}
\Delta \phi+W \phi+R+N(\phi)=0 & \text { in } \Omega_{\varepsilon} \\
\partial \phi / \partial \nu=0 & \text { on } \partial \Omega_{\varepsilon}
\end{aligned}\right.
$$

where

(2-18) $\quad W=2 \varepsilon^{4} \cosh V$,

(2-19) $\quad N(\phi)=2 \varepsilon^{4}(\sinh (V+\phi)-\phi \cosh V-\sinh V) \quad$ (the nonlinear term),

(2-20) $\quad R=\Delta V+2 \varepsilon^{4} \sinh V$ (the error term).

We choose the parameters $\mu_{i}$ as

$$
\log \left(8 \mu_{i}^{2}\right)=H\left(\xi_{i}, \xi_{i}\right)+\sum_{j \neq i}(-1)^{j+i} G\left(\xi_{i}, \xi_{j}\right)
$$


From Appendix A, we have for all $y \in \Omega_{\varepsilon}$ the estimates

$$
\begin{aligned}
|R(y)| & \leq C \varepsilon^{\alpha} \sum_{i=1}^{2(k+l)} \frac{1}{1+\left|y-\xi_{i}^{\prime}\right|^{3}}, \\
W(y) & =\sum_{i=1}^{2(k+l)} \frac{8 \mu_{i}^{2}}{\left(\mu_{i}^{2}+\left|y-\xi_{i}^{\prime}\right|^{2}\right)^{2}}\left(1+\theta_{\varepsilon}(y)\right),
\end{aligned}
$$

with

$$
\left|\theta_{\varepsilon}(y)\right| \leq C \varepsilon^{\alpha}+C \varepsilon \sum_{i=1}^{2(k+l)}\left|y-\xi_{i}^{\prime}\right|,
$$

where $\xi_{i}^{\prime}=\xi_{i} / \varepsilon$.

\section{Analysis of the linearized problem}

In this section we study the solvability of the problem

$$
\left\{\begin{aligned}
-\Delta \phi & =W \phi+h+\sum_{i=1}^{2(k+l)} \sum_{j=1}^{J_{i}} c_{j i} \chi_{i} Z_{j i}+c_{0} \chi Z & & \text { in } \Omega_{\varepsilon}, \\
\frac{\partial \phi}{\partial v} & =0 & & \text { on } \partial \Omega_{\varepsilon}
\end{aligned}\right.
$$

with

$$
\begin{aligned}
\int_{\Omega_{\varepsilon}} \chi_{i} Z_{j i} \phi & =0 \quad \text { for } i=1, \ldots, 2(k+l), j=1, J_{i}, \\
\int_{\Omega_{\varepsilon}} \chi Z \phi & =0,
\end{aligned}
$$

where $W$ is a function that satisfies (2-23) and (2-24), $h \in L^{\infty}\left(\Omega_{\varepsilon}\right), c_{0}, c_{j i} \in \mathbb{R}$, the functions $\chi, \chi_{i}, Z$ and $Z_{j i}$ will be defined below, $J_{i}=2$ for $i=1, \ldots, 2 k$, and $J_{i}=1$ for $i=2 k+1, \ldots, 2(k+l)$.

Define $z_{j i}$ by

$$
z_{0 i}=\frac{1}{\mu_{i}}-2 \frac{\mu_{i}}{\mu_{i}^{2}+|y|^{2}} \quad \text { and } \quad z_{j i}=\frac{y_{j}}{\mu_{i}^{2}+|y|^{2}} .
$$

It is well known that any solution to

$$
\Delta \phi+\frac{8 \mu_{i}^{2}}{\left(\mu_{i}^{2}+|y|^{2}\right)^{2}} \phi=0, \quad|\phi| \leq C(1+|y|)^{\sigma}
$$

is a linear combination of $z_{j i}$ for $j=0,1,2$; see [Chen and Lin 2002, Lemma 2.1]. 
Next, we fix a large constant $R_{0}$ and a nonnegative smooth function $\bar{\chi}: \mathbb{R} \rightarrow \mathbb{R}$ such that $\bar{\chi}(r)=1$ for $r \leq R_{0}, \bar{\chi}(r)=0$ for $r>R_{0}+1$, and $0 \leq \bar{\chi} \leq 1$.

For $i=1, \ldots, 2 k$ (corresponding to the interior bubble case), we define

$$
\chi_{i}(y)=\bar{\chi}\left(\left|y-\xi_{i}^{\prime}\right|\right), \quad Z_{j i}(y)=z_{j i}\left(y-\xi_{i}^{\prime}\right) \quad \text { for } j=0,1,2, i=1, \ldots, 2 k .
$$

For $i=2 k+1, \ldots, 2(k+l)$ (corresponding to the boundary bubble case), first we strength the boundary similarly to [del Pino and Wei 2006]. Let us concentrate on $\xi_{i} \in \partial \Omega$. Without loss of generality, we assume that $\xi_{i}=0$ and the unit outward normal at $\xi_{i}$ is $(0,-1)$. Let $P\left(x_{1}\right)$ be the defining function for the boundary $\partial \Omega$ in a neighborhood $B_{\rho}\left(\xi_{i}\right)$, that is,

$$
\Omega \cap B_{\rho}\left(\xi_{i}\right)=\left\{\left(x_{1}, x_{2}\right) \mid x_{2}>P\left(x_{1}\right),\left(x_{1}, x_{2}\right) \in B_{\rho}\left(\xi_{i}\right)\right\},
$$

and then define $F_{i}: B_{\rho}\left(\xi_{i}\right) \cap \mathcal{N} \rightarrow \mathbb{R}^{2}$ by $F_{i}=\left(F_{i 1}, F_{i 2}\right)$, where

$$
F_{i 1}=x_{1}+\frac{x_{2}-P\left(x_{1}\right)}{1+\left|P^{\prime}\left(x_{1}\right)\right|^{2}} P^{\prime}\left(x_{1}\right) \quad \text { and } \quad F_{i 2}=x_{2}-P\left(x_{1}\right) .
$$

Then we set

$$
F_{i}^{\varepsilon}(y)=\varepsilon^{-1} F_{i}(\varepsilon y)
$$

and define

$\chi_{i}(y)=\bar{\chi}\left(F_{i}^{\varepsilon}(y)\right), \quad Z_{j i}(y)=z_{j i}\left(F_{i}^{\varepsilon}(y)\right) \quad$ for $j=0,1, i=2 k+1, \ldots, 2(k+l)$.

It is important to observe that $F_{i}$ preserves the Neumann boundary condition and

$$
\Delta Z_{0 i}+\frac{8 \mu_{i}}{\left(\mu_{i}^{2}+\left|y-\xi_{i}^{\prime}\right|^{2}\right)^{2}} Z_{0 i}=O\left(\frac{\varepsilon^{\alpha}}{\left(1+\left|y-\xi_{i}^{\prime}\right|\right)^{3}}\right) .
$$

Let $0<b<1$ and define for all $i=1, \ldots, 2(k+l)$,

$$
Z(y)=\left\{\begin{array}{cc}
\min \left\{1 / \mu_{i}-\varepsilon^{b}, Z_{0 i}(y)\right\} & \text { if }\left|y-\xi_{i}^{\prime}\right|<\delta / \varepsilon \\
1 / \mu_{i}-\varepsilon^{b} & \text { if }\left|y-\xi_{i}^{\prime}\right| \geq \delta / \varepsilon
\end{array}\right.
$$

and $\chi=\sum_{i=1}^{2(k+l)} \chi_{i}$.

Now let us introduce the norms

$$
\|h\|_{\infty}=\sup _{y \in \Omega_{\varepsilon}}|h(y)| \quad \text { and } \quad\|h\|_{*}=\sup _{y \in \Omega_{\varepsilon}} \frac{|h(y)|}{\varepsilon^{2}+\sum_{i=1}^{2(k+l)}\left(1+\left|y-\xi_{i}^{\prime}\right|\right)^{-2-\sigma}},
$$

where we fix $0<\sigma<1$, reserving the precise choice for later. Our main result in this section is stated as follows:

Proposition 3.1. Let $d>0$ and let $k, l$ be nonnegative integers with $k+l \geq$ 1. Then there exists a $\varepsilon_{0}$ such that for any $0<\varepsilon<\varepsilon_{0}$, any $2(k+l)$-points $\left(\xi_{1}, \ldots, \xi_{2(k+l)}\right) \in M_{d}$ and any $h \in L^{\infty}\left(\Omega_{\varepsilon}\right)$, there is a unique solution $\phi \in L^{\infty}\left(\Omega_{\varepsilon}\right)$, 
$c_{0}, c_{j i} \in \mathbb{R}$ to (3-1), with $i=1, \ldots, 2(k+l)$ and $j=1, J_{i}$. Moreover there is a positive $C$ independent of $\varepsilon$ such that

$$
\begin{aligned}
\|\phi\|_{L^{\infty}\left(\Omega_{\varepsilon}\right)} & \leq C|\log \varepsilon|\|h\|_{*}, \\
\max \left\{\left|c_{0}\right|,\left|c_{j i}\right|\right\} & \leq C\|h\|_{*} \quad \text { for } i=1, \ldots, 2(k+l), j=1, J_{i} .
\end{aligned}
$$

We begin to prove this result by studying a linear problem

$$
\left\{\begin{aligned}
-\Delta \phi & =h+W \phi & & \text { in } \Omega_{\varepsilon} \\
\partial \phi / \partial \nu & =0 & & \text { on } \partial \Omega_{\varepsilon}
\end{aligned}\right.
$$

together with orthogonality conditions (3-2) and (3-3).

Proposition 3.2. Let $h \in L^{\infty}\left(\Omega_{\varepsilon}\right)$. For fixed $d>0$ there exist $\varepsilon_{0}>0$ and $C$ such that if $0<\varepsilon<\varepsilon_{0}, \xi=\left(\xi_{1}, \ldots, \xi_{2(k+l)}\right) \in M_{d}$ and $\phi \in L^{\infty}\left(\Omega_{\varepsilon}\right)$ is a solution of (3-6) such that (3-2) and (3-3) hold, then

$$
\|\phi\|_{L^{\infty}\left(\Omega_{\varepsilon}\right)} \leq C \log \varepsilon^{-1}\|h\|_{*},
$$

where $C$ is independent of $\varepsilon$.

We will prove this estimate by contradiction assuming that there exist a sequence $\varepsilon \rightarrow 0$, points $\left(\xi_{1}, \ldots, \xi_{2(k+l)}\right) \in M_{d}$ (we omit the dependence on $\varepsilon$ in the notation) and functions $h, \phi \in L^{\infty}\left(\Omega_{\varepsilon}\right)$ such that

$$
\|\phi\|_{L^{\infty}\left(\Omega_{\varepsilon}\right)}=1 \quad \text { and } \quad \log \varepsilon^{-1}\|h\|_{*}=o(1) .
$$

Fix $0<\gamma<\beta<1 / 2$ and consider the function $\eta$ given by

$$
\eta(r)= \begin{cases}1 & \text { if } r<\varepsilon^{-\gamma}, \\ \frac{\log \varepsilon^{-\beta}-\log r}{\log \varepsilon^{-\beta}-\log \varepsilon^{-\gamma}} & \text { if } \varepsilon^{-\gamma}<r<\varepsilon^{-\beta}, \\ 0 & \text { if } r>\varepsilon^{-\beta} .\end{cases}
$$

Let $\tilde{\eta}$ be a radial smooth cut-off function on $\mathbb{R}^{2}$ such that $\tilde{\eta}(r) \equiv 1$ for $r<\varepsilon^{-\beta}$, $\tilde{\eta} \equiv 0$ for $r>2 \varepsilon^{-\beta},\left|\tilde{\eta}^{\prime}(r)\right| \leq C \varepsilon^{\beta}$ and $\left|\tilde{\eta}^{\prime \prime}(r)\right| \leq C \varepsilon^{2 \beta}$. Then we set

$$
\begin{aligned}
\eta_{1 i}(y) & = \begin{cases}\eta\left(\left|y-\xi_{i}^{\prime}\right|\right) & \text { for } i=1, \ldots, 2 k, \\
\eta\left(\left|F_{i}^{\varepsilon}(y)\right|\right) & \text { for } i=2 k+1, \ldots, 2(k+l) ;\end{cases} \\
\eta_{2 i}(y) & = \begin{cases}\tilde{\eta}\left(\left|y-\xi_{i}^{\prime}\right|\right) & \text { for } i=1, \ldots, 2 k, \\
\tilde{\eta}\left(\left|F_{i}^{\varepsilon}(y)\right|\right) & \text { for } i=2 k+1, \ldots, 2(k+l) ;\end{cases} \\
a_{0 i} & =\frac{1}{\mu_{i}\left(\left(4 / c_{i}\right) \log \varepsilon^{\gamma-1}+H\left(\xi_{i}, \xi_{i}\right)\right)}
\end{aligned}
$$

and also

$$
\widehat{Z}_{0 i}(y)=Z_{0 i}(y)-\mu_{i}^{-1}+a_{0 i} G\left(\varepsilon y, \xi_{i}\right) .
$$


Now define a test function

$$
\tilde{Z}_{0 i}=\eta_{1 i} Z_{0 i}+\varepsilon\left(1-\eta_{1 i}\right) \eta_{2 i} \widehat{Z}_{0 i} .
$$

Given $\phi$ satisfying (3-6) and the orthogonality conditions (3-2) and (3-3), let

$$
\tilde{\phi}=\phi-\sum_{i=1}^{2(k+l)} d_{i} \tilde{Z}_{0 i}
$$

where the numbers $d_{i}$ are chosen so that $\int_{\Omega_{\varepsilon}} \chi_{i} Z_{0 i} \tilde{\phi}=0$ for any $i=1, \ldots, 2(k+l)$, namely $d_{i}=\int_{\Omega_{\varepsilon}} \chi_{i} Z_{0 i} \phi / \int_{\Omega_{\varepsilon}} \chi_{i} Z_{0 i}^{2}$. Observe that

$$
d_{i}=O(1) \quad \text { and } \quad\|\tilde{\phi}\|_{L^{\infty}\left(\Omega_{\varepsilon}\right)}=O(1) .
$$

Moreover, $\tilde{\phi}$ satisfies

$$
\begin{cases}-\Delta \tilde{\phi}=W \tilde{\phi}+h-\sum_{i=1}^{2(k+l)} d_{i} L\left(\tilde{Z}_{0 i}\right) & \text { in } \Omega_{\varepsilon}, \\ \partial \tilde{\phi} / \partial v=0 & \text { on } \partial \Omega_{\varepsilon}\end{cases}
$$

and the orthogonality condition

$$
\int_{\Omega_{\varepsilon}} \chi_{i} Z_{j i} \tilde{\phi}=0 \quad \text { for all } i=1, \ldots, 2(k+l), j=0,1, J_{i},
$$

where $L:=-\Delta-W$.

To reach a contradiction it is sufficient to establish the following:

Lemma 3.3. $\tilde{\phi} \rightarrow 0$ uniformly in $\Omega_{\varepsilon}$.

Lemma 3.4. $d_{i} \rightarrow 0$ for all $i=1, \ldots, 2(k+l)$.

We postpone proofs of these lemmas and mention first some key steps.

Lemma 3.5. For all $i=1, \ldots, 2(k+l)$ and $R>0$, we have

$$
\tilde{\phi} \rightarrow 0 \text { uniformly in } \Omega_{\varepsilon} \cap B_{R}\left(\xi_{i}^{\prime}\right) .
$$

Proof. Assume that for some $R>0$ and $i=1, \ldots, 2(k+l)$ there is a $c>0$ such that $\sup _{B_{R}\left(\xi_{i}^{\prime}\right)}|\tilde{\phi}| \geq c>0$ for a subsequence $\varepsilon \rightarrow 0$. Let us translate and rotate $\Omega_{\varepsilon}$ so that $\xi_{i}^{\prime}=0$ and $\Omega_{\varepsilon}$ approaches the upper half plane $\mathbb{R}_{+}^{2}$. By the elliptic estimate, $\tilde{\phi} \rightarrow \tilde{\phi}_{0}$ uniformly on compact sets and $\tilde{\phi}_{0}$ is a nontrivial bounded solution of (3-4). Then we conclude that $\tilde{\phi}_{0}$ is a linear combination of $z_{j i}$ for $j=0,1, J_{i}$. On the other hand, we can take the limit in the orthogonality relations (3-10), observing that the limits of the functions $Z_{j i}$ are just rotations and translations of $z_{j i}$, and we find that $\int_{\mathbb{R}_{+}^{2}} \chi \tilde{\phi}_{0} z_{j i}=0$. This contradicts the fact that $\tilde{\phi}_{0} \not \equiv 0$. 
Lemma 3.6. $\overline{\tilde{\phi}} \equiv \frac{1}{\left|\Omega_{\varepsilon}\right|} \int_{\Omega_{\varepsilon}} \tilde{\phi} \rightarrow 0$.

Proof. By potential theory we have

$$
\tilde{\phi}(y)-\overline{\tilde{\phi}}=\int_{\Omega_{\varepsilon}} G(\varepsilon y, \varepsilon z)\left(W \tilde{\phi}+h-\sum_{i=1}^{2(k+l)} d_{i} L\left(\tilde{Z}_{0 i}\right)\right) d z,
$$

where $G$ is the Green function defined by (1-3).

Note that since

$$
\int_{\Omega_{\varepsilon}} W \tilde{\phi}+h-\sum_{i=1}^{2(k+l)} d_{i} L\left(\tilde{Z}_{0 i}\right)=0
$$

and

$$
G(\varepsilon y, \varepsilon z)=-\frac{4}{c_{i}} \log \varepsilon-\frac{4}{c_{i}} \log |y-z|+H(\varepsilon y, \varepsilon z),
$$

we have

$$
\begin{aligned}
& \tilde{\phi}(y)-\overline{\tilde{\phi}} \\
& =\frac{1}{8 \pi} \int_{\Omega_{\varepsilon}}\left(H(\varepsilon y, \varepsilon z)-\frac{4}{c_{i}} \log |y-z|\right)\left(W \tilde{\phi}+h-\sum_{i=1}^{2(k+l)} d_{i} L\left(\tilde{Z}_{0 i}\right)\right) d z .
\end{aligned}
$$

Since $\tilde{\phi}(y) \rightarrow 0$ uniformly on sets of the form $\left|y-\xi_{i}^{\prime}\right|<R$, we can select a sequence $R_{\varepsilon} \rightarrow \infty$ such that

$$
\tilde{\phi}(y) \rightarrow 0 \quad \text { uniformly for }\left|y-\xi_{i}^{\prime}\right|<R_{\varepsilon} .
$$

We can assume $R_{\varepsilon} \rightarrow \infty$ as slowly as we need.

Select a point $y_{m} \in \Omega_{\varepsilon}$ for $m=1, \ldots, 2 k$ or $y_{m} \in \partial \Omega_{\varepsilon}$ for $m=2 k+1, \ldots, 2(k+l)$, such that $\left|y_{m}-\xi_{m}^{\prime}\right|=R_{\varepsilon}$. We claim that when we evaluate (3-11) at $y_{m}$, all terms in the right side of (3-11) converge to zero except for

$$
\int_{\Omega_{\varepsilon}} \log \left|y_{m}-z\right| L\left(\tilde{Z}_{0 i}\right) d z=\frac{2 \pi}{\mu_{i}} \delta_{m i}+o(1),
$$

where $\delta_{m i}$ is Kronecker's delta.

Claim 1. $\int_{\Omega_{\varepsilon}} \log \left|y_{m}-z\right| L\left(\tilde{Z}_{0 i}\right) d z=\frac{2 \pi}{\mu_{i}} \delta_{m i}+o(1)$.

This is proved in Appendix B.

Claim 2. $\int_{\Omega_{\varepsilon}} \log |y-z| h(z) d z=o(1) \quad$ uniformly for $y \in \Omega_{\varepsilon}$. 
Proof. Observe that $\log |y-z|=O\left(\log \varepsilon^{-1}\right)$ for $|y-z|>R$, where $R>0$ is fixed, and that $\int_{\Omega_{\varepsilon} \cap B_{R}(y)}|\log | y-z|| d z \leq C$. Then

$$
\left|\int_{\Omega_{\varepsilon}} \log \right| y-z|h d z| \leq C \log \varepsilon^{-1}\|h\|_{*}=o(1) .
$$

Claim 3. $\int_{\Omega_{\varepsilon}} \log |y-z| W \tilde{\phi} d z=o(1)$.

Proof. It suffices to show that $\log \varepsilon^{-1} \int_{\Omega_{\varepsilon}} W \tilde{\phi} d z=o(1)$. Integrating equation (3-9), we have

$$
\int_{\Omega_{\varepsilon}} W \tilde{\phi}+h-\sum_{i=1}^{2(k+l)} d_{i} L\left(\tilde{Z}_{0 i}\right)=0 .
$$

The claim then follows from (B-10) and (3-7).

Claim 4. $A \equiv \int_{\Omega_{\varepsilon}} H(\varepsilon y, \varepsilon z)\left(W \tilde{\phi}+h-L\left(\tilde{Z}_{0 i}\right)\right)=o(1) \quad$ uniformly for $y \in \Omega_{\varepsilon}$.

This is proved in Appendix B.

We now return to the proof of Lemma 3.6. From claims above, we get

$$
\tilde{\phi}\left(y_{i}\right)-\overline{\tilde{\phi}}=\frac{8 \pi d_{i}}{c_{i} \mu_{i}}+o(1) \quad \text { for all } i=1, \ldots, 2(k+l) .
$$

But the orthogonality condition (3-3) implies that

$$
\sum_{i=1}^{2(k+l)} d_{i} a_{i}=0, \quad \text { where } a_{i}=\int_{\Omega_{\varepsilon}} \chi_{i} Z_{0 i}^{2}>0 .
$$

Multiplying (3-12) by $c_{i} a_{i} \mu_{i}$, adding and using (3-13), we find

$$
\sum_{i=1}^{2(k+l)} c_{i} \mu_{i} a_{i} \tilde{\phi}\left(y_{i}\right)-a \overline{\tilde{\phi}}=o(1), \quad \text { where } a=\sum_{i=1}^{2(k+l)} c_{i} a_{i} \mu_{i} .
$$

Since $\tilde{\phi}\left(y_{i}\right) \rightarrow 0$ and $a$ is bounded away from zero, we get that $\overline{\tilde{\phi}}=o(1)$.

Proof of Lemma 3.3. Let $\check{\phi}=\tilde{\phi}(x / \varepsilon)$, with $x \in \Omega$. Then $\check{\phi}$ satisfies

$$
\left\{\begin{aligned}
-\Delta \check{\phi}(x) & =\varepsilon^{-2}\left(\check{W} \check{\phi}+h+\sum_{i=1}^{2(k+l)} d_{i}\left(\Delta \check{Z}_{0 i}+\check{W} \check{Z}_{0 i}\right)\right) & & \text { in } \Omega, \\
\partial \check{\phi} / \partial \nu & =0 & & \text { on } \partial \Omega,
\end{aligned}\right.
$$

where $\check{W}(x)=W(x / \varepsilon), \check{Z}_{0 i}(x)=\tilde{Z}_{0 i}(x / \varepsilon)$ and $\check{h}(x)=h(x / \varepsilon)$. For given $\delta>0$, let $E_{\delta}=\Omega \backslash \bigcup_{i=1}^{2(k+l)} B_{\delta}\left(\xi_{i}\right)$. Then

$$
\frac{1}{\varepsilon^{2}}\|\check{h}\|_{L^{\infty}\left(E_{\delta}\right)} \leq C\|h\|_{*} \rightarrow 0 \quad \text { and } \quad \frac{1}{\varepsilon^{2}}\|\check{W} \check{\phi}\|_{L^{\infty}\left(E_{\delta}\right)} \leq C \varepsilon^{2} .
$$


Furthermore, in $E_{\delta}$ we have $\check{Z}_{0 i} \equiv 0$. Recalling $\|\check{\phi}\|_{L^{\infty}(\Omega)} \leq 1$ and $|\Omega|^{-1} \int_{\Omega} \check{\phi} \rightarrow 0$, we obtain $\breve{\phi} \rightarrow 0$ uniformly in $E_{\delta}$ and this implies

$$
\tilde{\phi} \rightarrow 0 \quad \text { uniformly in } \Omega_{\varepsilon} \backslash \bigcup_{i=1}^{2(k+l)} B_{\delta / \varepsilon}\left(\xi_{i}^{\prime}\right) \text { for any } \delta>0 .
$$

For a given $R_{1}>0$, let $A_{i}=B_{\delta / \varepsilon}\left(\xi_{i}^{\prime}\right) \backslash B_{R_{1}}\left(\xi_{i}^{\prime}\right)$. Given $\varepsilon>0$ small enough, there exist $R_{1}>1$ independent of $\varepsilon$ (if necessary we can choose $R_{1}$ large enough) and $\psi_{i}: \Omega_{\varepsilon} \cap A_{i} \rightarrow \mathbb{R}$ smooth and positive such that

$$
\left\{\begin{aligned}
-\Delta \psi_{i}-W \psi_{i} & \geq C\left|y-\xi_{i}^{\prime}\right|^{-2-\sigma}+\varepsilon^{2} & & \text { in } \Omega_{\varepsilon} \cap A_{i}, \\
\partial \psi_{i} / \partial \nu & \geq 0 & & \text { on } \partial \Omega_{\varepsilon} \cap A_{i}, \\
\psi_{i} & >0 & & \text { in } \Omega_{\varepsilon} \cap A_{i}, \\
\psi_{i} \geq c & >0 & & \text { on } \partial A_{i} \cap \Omega_{\varepsilon},
\end{aligned}\right.
$$

where $C, c>0$ can be chosen independent of $\varepsilon$ and $\psi_{i}$ is bounded uniformly in $\Omega_{\varepsilon} \cap A_{i}$. Let $\Psi_{0}$ be the unique solution of

$$
\Delta \Psi_{0}-\varepsilon^{4} \Psi_{0}+\varepsilon^{2}=0 \quad \text { in } \Omega_{\varepsilon}, \quad \partial \Psi_{0} / \partial \nu=\varepsilon \quad \text { on } \partial \Omega_{\varepsilon},
$$

and take $\psi_{1 i}=1-r^{-\sigma}$, where $r=\left|y-\xi_{i}^{\prime}\right|$. Then we claim that the function

$$
\psi_{i}(y)=\frac{4}{\sigma^{2}}\left(C \Psi_{0}+\psi_{1 i}\right)
$$

satisfies the requirements.

In fact, a simple calculation shows that

$$
-\Delta \psi_{1 i}=\sigma^{2} r^{-2-\sigma} \text {. }
$$

If $\xi_{i}^{\prime} \in \Omega_{\varepsilon}$, we have

$$
\frac{\partial \psi_{1 i}}{\partial v_{\varepsilon}}=O\left(\varepsilon^{1+\sigma}\right) \quad \text { on } \partial \Omega_{\varepsilon}
$$

If $\xi_{i}^{\prime} \in \partial \Omega_{\varepsilon}$ and $\left|y-\xi_{i}^{\prime}\right|>R$, we have

$$
\frac{\partial \psi_{1 i}}{\partial v_{\varepsilon}}=\sigma \frac{\left(y-\xi_{i}^{\prime}\right) \cdot v_{\varepsilon}}{r^{2+\sigma}} \quad \text { on } \partial \Omega_{\varepsilon}
$$

As before, we write $\partial \Omega_{\varepsilon}$ near $\xi_{i}^{\prime}$ as the graph $\left\{\left(y_{1}, y_{2}\right) \mid y_{2}=\varepsilon^{-1} P\left(\varepsilon y_{1}\right)\right\}$ with $P(0)=P^{\prime}(0)=0$. Then we have

$$
\frac{\partial \psi_{1 i}}{\partial v_{\varepsilon}}=\frac{\sigma}{r^{2+\sigma}} \frac{y_{1} P^{\prime}\left(\varepsilon y_{1}\right)-P\left(\varepsilon y_{1}\right)}{\sqrt{1+P^{\prime}\left(\varepsilon y_{1}\right)^{2}}}=\frac{\sigma}{r^{2+\sigma}} \frac{O\left(\varepsilon r^{2}\right)}{\sqrt{1+O\left(\delta^{2}\right)}}=O\left(\frac{\varepsilon}{r^{\sigma}}\right)
$$

for all $R<r<\delta / \varepsilon$. Thus we see that

$$
\frac{\partial \psi_{1 i}}{\partial v_{\varepsilon}}=o(\varepsilon) \quad \text { on } \partial \Omega_{\varepsilon}
$$


Therefore, for $\left|y-\xi_{i}^{\prime}\right|>R$ with $i=1, \ldots, 2(k+l)$, where $R$ is large, we have by the definition of $\psi_{i}$ and the fact that $W \leq 1 /\left(1+\left|y-\xi_{i}^{\prime}\right|^{4}\right)$ that

$$
-\Delta \psi_{i}-W \psi_{i}=\frac{C}{\sigma^{2}}\left(\varepsilon^{2}-\varepsilon^{4} \Psi_{0}\right)-\frac{4}{\sigma^{2}} \frac{C \Psi_{0}+\psi_{1 i}}{1+r^{4}}+\frac{C}{r^{2+\sigma}} \geq \varepsilon^{2}+\frac{C}{r^{2+\sigma}} .
$$

And on $\partial \Omega_{\varepsilon}$,

$$
\frac{\partial \psi_{i}}{\partial \nu_{\varepsilon}} \geq C \varepsilon
$$

This verifies the claim.

Thanks to the barrier $\psi_{i}$, we deduce that the following maximum principle holds in $\Omega_{\varepsilon} \cap A_{i}$. If $\phi \in H^{1}\left(\Omega_{\varepsilon} \cap A_{i}\right)$ satisfies

$$
\left\{\begin{aligned}
-\Delta \phi-W \phi \geq 0 & \text { in } \Omega_{\varepsilon} \cap A_{i}, \\
\phi \geq 0 & \text { on } \partial \Omega_{\varepsilon} \cap A_{i},
\end{aligned}\right.
$$

then $\phi \geq 0$ in $\Omega_{\varepsilon} \cap A_{i}$.

Let $h$ be bounded and $\tilde{\phi}$ be a solution of (3-9) satisfying (3-10). We first claim that $\|\tilde{\phi}\|_{L^{\infty}\left(\Omega_{\varepsilon} \cap A_{i}\right)}$ can be controlled in terms of

$$
\sum_{i=1}^{2(k+l)}\left|d_{i}\right|\left\|L\left(\tilde{Z}_{0 i}\right)\right\|_{*}, \quad \sup _{\Omega_{\varepsilon} \cap \partial A_{i}}|\tilde{\phi}|, \quad \text { and } \quad\|h\|_{*} .
$$

Indeed, set

$$
\Phi=C\left(\sup _{\Omega_{\varepsilon} \cap \partial A_{i}}|\tilde{\phi}|+\|h\|_{*}+\sum_{i=1}^{2(k+l)}\left|d_{i}\right|\left\|L\left(\tilde{Z}_{0 i}\right)\right\|_{*}\right) \psi_{i} .
$$

By the maximum principle above, we have $|\tilde{\phi}| \leq \Phi$ in $\Omega_{\varepsilon} \cap A_{i}$. Since $\psi_{i}$ is uniformly bounded, we get

$$
|\tilde{\phi}| \leq C\left(\sup _{\Omega \varepsilon \cap \partial B_{R_{1}}\left(\xi_{i}^{\prime}\right)}|\tilde{\phi}|+\sup _{\Omega \varepsilon \cap \partial B_{\delta / \varepsilon}\left(\xi_{i}^{\prime}\right)}|\tilde{\phi}|+\|h\|_{*}+\sum_{i=1}^{2(k+l)}\left|d_{i}\right|\left\|L\left(\tilde{Z}_{0 i}\right)\right\|_{*}\right)
$$

in $\Omega_{\varepsilon} \cap A_{i}$. But $\|h\|_{*}=o(1)$ by the assumption, $\sup _{\Omega \varepsilon \cap \partial B_{R_{1}}}\left(\xi_{i}^{\prime}\right)|\tilde{\phi}| \rightarrow 0$ by Lemma 3.5, and $\sup _{\Omega \varepsilon \cap \partial B_{\delta / \varepsilon}\left(\xi_{i}^{\prime}\right)}|\tilde{\phi}| \rightarrow 0$ as shown above. At the same time, we also know $\left|d_{i}\right|=O(1)$ and $\left\|L\left(\tilde{Z}_{0 i}\right)\right\|_{*}=O\left(\varepsilon^{2 \gamma}\right)=o(1)$ from (B-10), this proves the result.

Proof of Lemma 3.4. We take $\tilde{Z}_{0 i}$ as test function to (3-9), obtaining

$$
\sum_{i=1}^{2(k+l)} d_{i} \int_{\Omega_{\varepsilon}} L\left(\tilde{Z}_{0 i}\right) \tilde{Z}_{0 i}=\int_{\Omega_{\varepsilon}} \tilde{\phi}\left(\Delta \tilde{Z}_{0 i}+W \tilde{Z}_{0 i}\right)+\int_{\Omega_{\varepsilon}} h \tilde{Z}_{0 i} .
$$


Observe that

$$
\left|\int_{\Omega_{\varepsilon}} \tilde{Z}_{0 i} h\right| \leq\|h\|_{*}\left\|\tilde{Z}_{0 i}\right\|_{L^{\infty}\left(\Omega_{\varepsilon}\right)} \leq C \log \varepsilon^{-1}\|h\|_{*} \frac{1}{\log \varepsilon^{-1}}=o(1) \frac{1}{\log \varepsilon^{-1}}
$$

and

$$
\left|\int_{\Omega_{\varepsilon}} \tilde{\phi}\left(\Delta \tilde{Z}_{0 i}+W \tilde{Z}_{0 i}\right)\right| \leq\|\tilde{\phi}\|_{L^{\infty}\left(\Omega_{\varepsilon}\right)}\left\|L\left(\tilde{Z}_{0 i}\right)\right\|_{*}=o(1) \frac{1}{\log \varepsilon^{-1}} .
$$

It is not difficult to show as above that

$$
\left|\int_{\Omega_{\varepsilon}} L\left(\tilde{Z}_{0 i}\right) \tilde{Z}_{0 i}\right| \geq \frac{C}{\log \varepsilon^{-1}} .
$$

Proof of Proposition 3.1. First we prove that for any $\phi, c_{j i}, c_{0}$ and any solution to (3-1), we have the bound

$$
\|\phi\|_{L^{\infty}\left(\Omega_{\varepsilon}\right)} \leq C \log \varepsilon^{-1}\|h\|_{*} .
$$

From Proposition 3.2, we obtain that

$$
\|\phi\|_{L^{\infty}\left(\Omega_{\varepsilon}\right)} \leq C \log \varepsilon^{-1}\left(\|h\|_{*}+\sum_{i=1}^{2(k+l)} \sum_{j=1}^{J_{i}}\left|c_{j i}\right|+\left|c_{0}\right|\right) .
$$

So it suffices to estimate the values of the constants $a_{j i}$ and $c_{0}$.

To this end, we multiple (3-1) by $Z_{j i}$ and integrate to find

$$
\int_{\Omega_{\varepsilon}} L(\phi) Z_{j i}=\int_{\Omega_{\varepsilon}} h Z_{j i}+c_{j i} \int_{\Omega_{\varepsilon}} \psi_{i} Z_{j i}^{2} .
$$

Note that $Z_{j i}=O\left(1 /\left(1+\left|y-\xi_{i}\right|\right)\right)$ for $j \neq 0$, so

$$
\int_{\Omega_{\varepsilon}} h Z_{j i}=O\left(\|h\|_{*}\right)
$$

and

$$
\int_{\Omega_{\varepsilon}} L(\phi) Z_{j i}=\int_{\Omega_{\varepsilon}} L\left(Z_{j i}\right) \phi+\int_{\partial \Omega_{\varepsilon}} \frac{\partial Z_{j i}}{\partial v} \phi=O\left(\varepsilon \log \varepsilon^{-1}\|\phi\|_{L^{\infty}\left(\Omega_{\varepsilon}\right)}\right) .
$$

Substituting (3-20) and (3-21) into (3-19), we obtain

$$
\left|C_{j i}\right|=O\left(\|h\|_{*}\right)+O\left(\varepsilon \log \varepsilon^{-1}\|\phi\|_{L^{\infty}\left(\Omega_{\varepsilon}\right)}\right) .
$$

On the other hand, multiplying (3-1) by $Z$ we get

$$
c_{0} \int_{\Omega_{\varepsilon}} \chi Z^{2}=\int_{\Omega_{\varepsilon}} L(\phi) Z-\int_{\Omega_{\varepsilon}} h Z .
$$


Estimating as before, we have

$$
\int_{\Omega_{\varepsilon}} h Z=O\left(\|h\|_{*}\right)
$$

and

$$
\int_{\Omega_{\varepsilon}} L(\phi) Z=\int_{\Omega_{\varepsilon}} L(Z) \phi=O\left(\varepsilon \log \varepsilon^{-1}\|\phi\|_{L^{\infty}\left(\Omega_{\varepsilon}\right)}\right) .
$$

Thus it follows from (3-23)-(3-25) that

$$
\left|c_{0}\right|=O\left(\|h\|_{*}\right)+O\left(\varepsilon \log \varepsilon^{-1}\|\phi\|_{L^{\infty}\left(\Omega_{\varepsilon}\right)}\right) .
$$

From (3-22) and (3-26) we see that the desired bound holds.

Now consider the Hilbert space

$$
H=\left\{\phi \in H^{1}\left(\Omega_{\varepsilon}\right): \int_{\Omega_{\varepsilon}} \chi Z \phi=0, \int_{\Omega_{\varepsilon}} \chi_{i} Z_{j i} \phi=0 \text { for } i=1, \ldots, 2(k+l), j=1, J_{i}\right\}
$$

with the norm $\|\phi\|_{H}^{2}=\int_{\Omega_{\varepsilon}}|\nabla \phi|^{2}$. Problem (3-1) is equivalent to finding $\phi \in H$ such that

$$
\int_{\Omega_{\varepsilon}} \nabla \phi \nabla \psi-\int_{\Omega_{\varepsilon}} W \phi \psi=\int_{\Omega_{\varepsilon}} h \psi \quad \text { for all } \psi \in H .
$$

By Fredholm's alternative, this is equivalent to the uniqueness of solutions to this problem, which is guaranteed by the a priori estimate (3-17).

Remark. The result of Proposition 3.1 implies that the unique solution $\phi=T(h)$ of (3-1) defines a continuous linear map from $L^{\infty}\left(\Omega_{\varepsilon}\right)$, with norm $\|\cdot\|_{*}$, into $L^{\infty}\left(\Omega_{\varepsilon}\right)$. Moreover, the operator $T$ is differential with respect to the variables $\xi_{m}^{\prime}$. In fact, computations similar to those used in [Wei et al. 2011] yield the estimate

$$
\left\|\partial_{\xi_{m}^{\prime}} \phi\right\|_{L^{\infty}\left(\Omega_{\varepsilon}\right)} \leq C\left(\log \varepsilon^{-1}\right)^{2}\|h\|_{*}
$$

\section{The nonlinear problem with constraints}

Let us introduce a small parameter $\tau$ and consider

$$
V_{1}(y)=V(y)+\tau Z(y) \text { for } y \in \Omega_{\varepsilon},
$$

where $V$ and $Z$ are given by (2-16) and (3-5). Then we set

$$
W_{1}=2 \varepsilon^{4} \cosh V_{1}, \quad R_{1}=\Delta V_{1}+2 \varepsilon^{4} \sinh V_{1}
$$

and

$$
N_{1}\left(\phi_{1}\right)=2 \varepsilon^{4}\left(\sinh \left(V_{1}+\phi_{1}\right)-\phi_{1} \cosh V_{1}-\sinh V_{1}\right)
$$


Now we consider the following auxiliary nonlinear problem:

$$
\left\{\begin{array}{l}
\Delta \phi_{1}+W_{1} \phi_{1}+R_{1}+N_{1}\left(\phi_{1}\right)+\sum_{i=1}^{2(k+l)} \sum_{j=1}^{J_{i}} c_{j i} \chi_{i} Z_{j i}+c_{0} \chi Z=0 \quad \text { in } \Omega_{\varepsilon}, \\
\int_{\Omega_{\varepsilon}} \chi Z \phi_{1} / \partial \nu=0 \quad \text { on } \partial \Omega_{\varepsilon}, \\
\text { for all } i=1, \ldots, 2(k+l), j=1, J_{\Omega_{\varepsilon}} \chi_{i} Z_{j i} \phi_{1}=0
\end{array}\right.
$$

Then we can follow the proofs [Wei et al. 2011, Lemma 4.1 and Theorem 4.2] to obtain the following results; we omit the details.

Lemma 4.1. Let $k+l \geq 1, d>0, \alpha \in(0,1)$ and $\tau=O\left(\varepsilon^{\theta}\right)$ with $\theta>\alpha / 2$. Then there exist $\varepsilon_{0}>0$ and $C>0$ such that for $0<\varepsilon<\varepsilon_{0}$ and for any $\xi_{1}, \ldots, \xi_{2(k+l)} \in M_{d}$, problem (4-2) admits a unique solution $\phi_{1}, c_{0}, c_{j i}$ for $i=1, \ldots, 2(k+l), j=1, J_{i}$, such that

$$
\left\|\phi_{1}\right\|_{L^{\infty}\left(\Omega_{\varepsilon}\right)} \leq C \varepsilon^{\alpha} .
$$

Furthermore, the function $\left(\tau, \xi^{\prime}\right) \rightarrow \phi_{1}\left(\tau, \xi^{\prime}\right) \in C\left(\bar{\Omega}_{\varepsilon}\right)$ is $C^{1}$ and

$$
\begin{aligned}
\left\|D_{\xi^{\prime}} \phi_{1}\right\|_{L^{\infty}\left(\Omega_{\varepsilon}\right)} \leq C|\log \varepsilon|^{2}\left(\varepsilon+\varepsilon^{2 \theta}+\varepsilon^{2 \alpha}\right), \\
\left\|D_{\tau} \phi_{1}\right\|_{L^{\infty}\left(\Omega_{\varepsilon}\right)} \leq C\left(\varepsilon^{\alpha}+\varepsilon^{\theta}\right)|\log \varepsilon| .
\end{aligned}
$$

Lemma 4.2. Let $k+l \geq 1$ and $d>0$. For any $0<\alpha<1$ there exist $\varepsilon_{0}>0$ and $C>0$ such that for $0<\varepsilon<\varepsilon_{0}$ and any $\left(\xi_{1}, \ldots, \xi_{2(k+l)}\right) \in M_{d}$, there exists a unique $\tau$ with $|\tau|=O\left(\varepsilon^{\alpha}\right)$ such that problem (4-2) admits a unique solution $\phi, c_{0}, c_{j i}$ for $i=1, \ldots, 2(k+l), j=1, J_{i}$ with $c_{0}=0$ and such that

$$
\|\phi\|_{L^{\infty}\left(\Omega_{\varepsilon}\right)} \leq C \varepsilon^{\alpha} .
$$

Furthermore, the function $\xi^{\prime} \mapsto \phi\left(\xi^{\prime}\right)$ is $C^{1}$ and

$$
\left\|D_{\xi^{\prime}} \phi\right\|_{L^{\infty}\left(\Omega_{\varepsilon}\right)} \leq C \varepsilon^{\alpha}|\log \varepsilon|^{2} .
$$

\section{Variational reduction and expansion of the energy}

In view of Lemmas 4.1 and 4.2 , given $\xi=\left(\xi_{1}, \ldots, \xi_{2(k+l)}\right) \in M_{d}$, we set $\phi(\xi)$ and $c_{j i}(\xi)$ to be the unique solution to (4-2) with $c_{0}=0$ satisfying the bounds (4-3) and (4-4). Let

$$
J_{\varepsilon}(v)=\frac{1}{2} \int_{\Omega_{\varepsilon}}|\nabla v|^{2} d x-2 \varepsilon^{4} \int_{\Omega_{\varepsilon}} \cosh v d x
$$

and define

$$
F_{\varepsilon}(\xi)=J_{\varepsilon}\left(V_{1}\left(\xi^{\prime}\right)+\phi\left(\xi^{\prime}\right)\right)
$$


where $\xi^{\prime}=\xi / \varepsilon$ and $V_{1}\left(\xi^{\prime}\right)=V\left(\xi^{\prime}\right)+\tau\left(\xi^{\prime}\right) Z\left(\xi^{\prime}\right)$ with $\tau(\xi)$ given by Lemma 4.2.

Lemma 5.1. If $\xi=\left(\xi_{1}, \ldots, \xi_{2(k+l)}\right) \in \mathcal{M}_{d}$ is a critical point of $F_{\varepsilon}$, then

$$
v=V_{1}\left(\xi^{\prime}\right)+\phi\left(\xi^{\prime}\right)
$$

is a critical point of $J_{\varepsilon}$, that is, a solution to (2-14).

Proof. A direct computation gives

$$
\frac{\partial F_{\varepsilon}}{\partial \xi_{m}}=\varepsilon^{-1} \frac{\partial J_{\varepsilon}\left(V_{1}\left(\xi^{\prime}\right)+\phi\left(\xi^{\prime}\right)\right)}{\partial \xi_{m}^{\prime}}=\varepsilon^{-1} D J_{\varepsilon}\left(V_{1}\left(\xi^{\prime}\right)+\phi\left(\xi^{\prime}\right)\right)\left(\frac{\partial V_{1}\left(\xi^{\prime}\right)}{\partial \xi_{m}^{\prime}}+\frac{\partial \phi\left(\xi^{\prime}\right)}{\partial \xi_{m}^{\prime}}\right) .
$$

Since $V_{1}\left(\xi^{\prime}\right)+\phi\left(\xi^{\prime}\right)$ solves (4-2) with $c_{0}=0$, we have

$$
\frac{\partial F_{\varepsilon}}{\partial \xi_{m}}=\varepsilon^{-1} \sum_{i=1}^{2(k+l)} \sum_{j=1}^{J_{i}} c_{j i} \int_{\Omega_{\varepsilon}} \chi_{i} Z_{j i}\left(\frac{\partial V_{1}\left(\xi^{\prime}\right)}{\partial \xi_{m}^{\prime}}+\frac{\partial \phi\left(\xi^{\prime}\right)}{\partial \xi_{m}^{\prime}}\right) .
$$

From the assumption $D F_{\varepsilon}(\xi)=0$, we obtain

$$
\sum_{i=1}^{2(k+l)} \sum_{j=1}^{J_{i}} c_{j i} \int_{\Omega_{\varepsilon}} \chi_{i} Z_{j i}\left(\frac{\partial V_{1}\left(\xi^{\prime}\right)}{\partial \xi_{m}^{\prime}}+\frac{\partial \phi\left(\xi^{\prime}\right)}{\partial \xi_{m}^{\prime}}\right)=0 \quad \text { for all } m=1, \ldots, 2(k+l) .
$$

Since

$$
\left\|\partial_{\xi_{m}^{\prime}} \phi\left(\xi^{\prime}\right)\right\|_{L^{\infty}\left(\Omega_{\varepsilon}\right)} \leq C \varepsilon^{\alpha}|\log \varepsilon|^{2} \quad \text { and } \quad \partial_{\xi_{m}^{\prime}} V\left(\xi^{\prime}\right)=(-1)^{m} Z_{j m}+o(1)
$$

for $j=1, J_{i}$, where $o(1)$ is in the $L^{\infty}$-norm as a direct consequence of (4-1), it follows that

$$
\sum_{i=1}^{2(k+l)} \sum_{j=1}^{J_{i}} c_{j i} \int_{\Omega_{\varepsilon}} \chi_{i} Z_{j i}\left((-1)^{m} Z_{j m}+o(1)\right)=0 \quad \text { for all } m=1, \ldots, 2(k+l),
$$

which is a strictly diagonal dominant system. This implies that $c_{j i}=0$ for all $i=1, \ldots, 2(k+l), j=1, J_{i}$.

A key step in seeking the critical points of the functional $F_{\varepsilon}$ is finding its expected closeness to the functional $J_{\varepsilon}\left(V_{1}(\xi)\right)$. The procedure is completely similar to that of [Wei et al. 2011, Theorem 5.2], so we omit it here.

Lemma 5.2. The expansion

$$
F_{\varepsilon}(\xi)=J_{\varepsilon}(V)+\theta_{\varepsilon}(\xi)
$$

holds with $\left|\theta_{\varepsilon}(\xi)\right|+\left|\nabla \theta_{\varepsilon}(\xi)\right|=o(1)$ uniformly on points in $\mathcal{M}_{d}$.

Now we will give an asymptotic estimate of $J_{\varepsilon}(V)$, where $V$ is defined by (2-16) and $J_{\varepsilon}$ is given as above. 
Lemma 5.3. Let $k+l \geq 1$, let $d>0$, let $\mu_{i}$ be given by (2-21) and let $V$ be the function defined in (2-16). Then the expansion

$$
\begin{aligned}
J_{\varepsilon}(V)=-\frac{1}{2} \sum_{i=1}^{2(k+l)} c_{i}\left(c_{i} H\left(\xi_{i}, \xi_{i}\right)+\sum_{j, j \neq i}(-1)^{j+i} c_{j} G\left(\xi_{j}, \xi_{i}\right)\right) \\
+2 \sum_{i=1}^{2(k+l)} c_{i} \log \varepsilon^{-1}+\sum_{i=1}^{2(k+l)} c_{i}(\log 8-2)+O\left(\varepsilon^{\alpha}\right) .
\end{aligned}
$$

holds uniformly on points $\xi=\left(\xi_{1}, \ldots, \xi_{2(k+l)}\right) \in \mathcal{M}_{d}$.

Proof. Recall the definition of $V(y)=\sum_{i=1}^{2(k+l)}(-1)^{i-1}\left(u_{i}(\varepsilon y)+H_{i}^{\varepsilon}(\varepsilon y)\right)$. We find that it satisfies

$$
\begin{cases}-\Delta V=\varepsilon^{4} \sum_{i=1}^{2(k+l)}(-1)^{i-1}\left(e^{u_{i}(\varepsilon y)}-\frac{1}{\left|\Omega_{\varepsilon}\right|} \int_{\Omega_{\varepsilon}} e^{u_{i}(\varepsilon y)}\right) & \text { in } \Omega_{\varepsilon}, \\ \partial V / \partial \nu=0 & \text { on } \partial \Omega_{\varepsilon} .\end{cases}
$$

We will compute the two terms in $J_{\varepsilon}(V)$.

First, by (5-3) we have

$$
\begin{aligned}
\int_{\Omega_{\varepsilon}}|\nabla V|^{2}=\int_{\Omega_{\varepsilon}}(-\Delta V) V & \\
= & \int_{\Omega_{\varepsilon}}\left(\varepsilon^{4} \sum_{j=1}^{2(k+l)}(-1)^{j-1}\left(e^{u_{j}(\varepsilon y)}-\frac{1}{\left|\Omega_{\varepsilon}\right|} \int_{\Omega_{\varepsilon}} e^{u_{j}(\varepsilon y)}\right)\right) \\
& \times\left(\sum_{i=1}^{2(k+l)}(-1)^{i-1}\left(u_{i}(\varepsilon y)+H_{i}^{\varepsilon}(\varepsilon y)\right)\right) \\
= & \varepsilon^{4} \sum_{j, i}(-1)^{j+i} \int_{\Omega_{\varepsilon}}\left(u_{i}(\varepsilon y)+H_{i}^{\varepsilon}(\varepsilon y)\right) e^{u_{j}(\varepsilon y)} \\
& -\frac{\varepsilon^{4}}{\left|\Omega_{\varepsilon}\right|}\left(\sum_{j=1}^{2(k+l)}(-1)^{j-1} \int_{\Omega_{\varepsilon}} e^{u_{j}(\varepsilon y)}\right)\left(\int_{\Omega_{\varepsilon}}^{2(k+l)} \sum_{i=1}^{2}(-1)^{i-1}\left(u_{i}(\varepsilon y)+H_{i}^{\varepsilon}(\varepsilon y)\right)\right) \\
= & \varepsilon^{4} \sum_{j, i}(-1)^{j+i} \int_{\Omega_{\varepsilon}}\left(u_{i}(\varepsilon y)+H_{i}^{\varepsilon}(\varepsilon y)\right) e^{u_{j}(\varepsilon y)}+O(\varepsilon),
\end{aligned}
$$

where the last equality is due to the fact $\varepsilon^{4} \sum_{j=1}^{2(k+l)}(-1)^{j-1} \int_{\Omega_{\varepsilon}} e^{u_{j}(\varepsilon y)}=O\left(\varepsilon^{4}\right)$, which can be easily deduced from (2-7). 
For $j \neq i$, we have by a calculation similar to (2-23)

$$
\begin{aligned}
\int_{\Omega_{\varepsilon}} \varepsilon^{4} & \left(u_{i}(\varepsilon y)+H_{i}^{\varepsilon}(\varepsilon y)\right) e^{u_{j}(\varepsilon y)} \\
& =\left(\int_{\Omega_{\varepsilon}^{1}}+\int_{\Omega_{\varepsilon}^{2}}\right)\left(\varepsilon^{4}\left(u_{i}(\varepsilon y)+H_{i}^{\varepsilon}(\varepsilon y)\right) e^{u_{j}(\varepsilon y)}\right) \\
& =\int_{\left.\Omega_{\varepsilon}^{1}\right|_{\xi_{j}^{\prime}}=0} \frac{8}{\left(1+y^{2}\right)^{2}}\left(\log \left|\xi_{i}-\xi_{j}\right|^{-4}+c_{i} H\left(\xi_{j}, \xi_{i}\right)\right)+O\left(\varepsilon^{\alpha}\right) \\
& =c_{j} c_{i} G\left(\xi_{j}, \xi_{i}\right)+O\left(\varepsilon^{\alpha}\right) .
\end{aligned}
$$

where $\Omega_{\varepsilon}^{1}:=B_{\delta /\left(\varepsilon \mu_{j}\right)}\left(\xi_{j}^{\prime}\right) \cap\left(\Omega_{\varepsilon} / \mu_{i}\right)$ and $\Omega_{\varepsilon}^{2}:=\left(\Omega_{\varepsilon} / \mu_{i}\right) \backslash \Omega_{\varepsilon}^{1}$. For $j=i$, we have

$$
\begin{aligned}
& \varepsilon^{4} \int_{\Omega_{\varepsilon}}\left(u_{i}(\varepsilon y)+H_{i}^{\varepsilon}(\varepsilon y)\right) e^{u_{i}(\varepsilon y)} \\
&= \int_{\Omega_{\varepsilon}} \frac{8 \mu_{i}^{2}}{\left(\mu_{i}^{2}+\left|y-\xi_{i}^{\prime}\right|^{2}\right)^{2}}\left(\log \frac{8 \mu_{i}^{2}}{\left(\varepsilon^{2} \mu_{i}^{2}+\left|\varepsilon y-\xi_{i}\right|^{2}\right)^{2}}+c_{i} H\left(\xi_{i}, \xi_{i}\right)\right. \\
&\left.\quad-\log \left(8 \mu_{i}^{2}\right)+O\left(\varepsilon^{\alpha}\right)+O\left(\varepsilon\left|y-\xi_{i}^{\prime}\right|\right)\right) \\
&=4 c_{i} \log \varepsilon^{-1}+c_{i}\left(c_{i} H\left(\xi_{i}, \xi_{i}\right)-2 \log 8 \mu_{i}^{2}\right)+2 c_{i}(\log 8-1)+O\left(\varepsilon^{\alpha}\right) .
\end{aligned}
$$

So from the choice of $\mu_{i}$ (see (2-21)), we get

$$
\begin{aligned}
\varepsilon^{4} \int_{\Omega_{\varepsilon}}\left(u_{i}(\varepsilon y)+\right. & \left.H_{i}^{\varepsilon}(\varepsilon y)\right) e^{u_{i}(\varepsilon y)}=4 c_{i} \log \varepsilon^{-1}+2 c_{i}(\log 8-1) \\
& -c_{i}\left(c_{i} H\left(\xi_{i}, \xi_{i}\right)+2 \sum_{m, m \neq i}(-1)^{m+i} c_{m} G\left(\xi_{m}, \xi_{i}\right)\right)+O\left(\varepsilon^{\alpha}\right) .
\end{aligned}
$$

Combining (5-4) and (5-5), we have

(5-6) $\frac{1}{2} \int_{\Omega_{\varepsilon}}|\nabla V|^{2}=-\frac{1}{2} \sum_{i=1}^{2(k+l)} c_{i}\left(c_{i} H\left(\xi_{i}, \xi_{i}\right)+\sum_{j, j \neq i}(-1)^{j+i} c_{j} G\left(\xi_{j}, \xi_{i}\right)\right)$

$$
+2 \sum_{i=1}^{2(k+l)} c_{i} \log \varepsilon^{-1}+(\log 8-1) \sum_{i=1}^{2(k+l)} c_{i}+O\left(\varepsilon^{\alpha}\right) .
$$

Next, let us compute the second term in $J_{\varepsilon}(V)$. Let $\Omega_{i}^{1}=B_{\delta / \varepsilon}\left(\xi_{i}^{\prime}\right) \cap\left(\Omega_{\varepsilon} / \mu_{i}\right)$. Then

$$
2 \varepsilon^{4} \int_{\Omega_{\varepsilon}} \cosh V=2 \varepsilon^{4} \sum_{i=1}^{2(k+l)} \int_{\Omega_{i}^{1}} \cosh V+O\left(\varepsilon^{2}\right) .
$$


Suppose first $i$ is odd. Then

$$
\begin{aligned}
2 \varepsilon^{4} \int_{\Omega_{i}^{1}} \cosh V & =\varepsilon^{4} \int_{\Omega_{i}^{1}} e^{V}+O(\varepsilon) \\
& =\int_{\Omega_{i}^{1}} \varepsilon^{4} e^{u_{i}(\varepsilon y)} \exp \left(H_{i}^{\varepsilon}+\sum_{m \neq i}(-1)^{m-1}\left(u_{m}+H_{m}^{\varepsilon}\right)\right)+O(\varepsilon) \\
& =c_{i}+O(\varepsilon) .
\end{aligned}
$$

Therefore

$$
2 \varepsilon^{4} \int_{\Omega_{i}^{1}} \cosh V=c_{i}+O(\varepsilon) .
$$

Similarly for $i$ even, we also have (5-7). So we obtain

$$
2 \varepsilon^{4} \int_{\Omega_{\varepsilon}} \cosh V=\sum_{i=1}^{2(k+l)} c_{i}+O(\varepsilon) .
$$

Finally, from (5-6) and (5-8) we conclude that (5-2) holds.

\section{Proof of main theorems}

Proof of Theorem 1.2. Let

$$
v(y)=V_{1}\left(\xi^{\prime}\right)(y)+\phi\left(\xi^{\prime}\right)(y) \text { for } y \in \Omega_{\varepsilon},
$$

where $V_{1}$ is given by (4-1) and $\phi$ is the unique solution to problem (4-2) with $c_{0}=0$, whose existence and properties are established in Lemma 4.2. According to Lemma 4.1, $v$ is a solution to problem (2-14) if we adjust $\xi$ so that it is a critical point of the function $F_{\varepsilon}(\xi)$ defined in (5-1), or equivalently, so that it is a critical point of

$$
\tilde{F}_{\varepsilon}(\xi)=2\left(2 \sum_{i=1}^{2(k+l)} c_{i} \log \varepsilon^{-1}+\sum_{i=1}^{2(k+l)} c_{i}(\log 8-2)-F_{\varepsilon}(\xi)\right) .
$$

From Lemmas 5.2 and 5.3 it follows that for $\xi \in \mathcal{M}_{d}$,

$$
\tilde{F}_{\varepsilon}(\xi)=\varphi_{2(k+l)}(\xi)+\varepsilon \Theta_{\varepsilon}(\xi)
$$

where $\Theta_{\varepsilon}$ and $\nabla_{\xi} \Theta_{\varepsilon}$ are uniformly bounded in the considered region as $\varepsilon \rightarrow 0$. On the other hand, $\tilde{F}_{\varepsilon} \rightarrow \varphi_{2(k+l)}$ uniformly on compact sets of $\mu_{d}$ as $\varepsilon$ goes to 0 . Now by Definition 1.1, we deduce that if $\varepsilon$ is small enough, there exists a critical point $\xi_{\varepsilon} \in \mathcal{M}_{d}$ of $\tilde{F}_{\varepsilon}$ such that $\tilde{F}_{\varepsilon} \rightarrow \varphi_{2(k+l)}\left(\xi^{*}\right)$. Moreover, up to subsequence, $\xi_{\varepsilon} \rightarrow \xi$ as $\varepsilon$ tends to 0 , with $\varphi_{2(k+l)}(\xi)=\varphi_{2(k+l)}\left(\xi^{*}\right)$. The function $u_{\varepsilon}(x)=v(y)$ is therefore 
a solution to (1-2) with the qualitative properties predicted by the theorem, as can be easily shown.

Proof of Theorem 1.3. First, we recall here some facts about the regular part of the Green function $H(x, y)$ defined by (1-4). If $y \in \Omega$ is a point close to $\partial \Omega$, we let $y^{*}$ be its uniquely determined reflection with respect to $\partial \Omega$. Now, we consider the auxiliary function

$$
H^{*}(x, y)=-\frac{1}{2 \pi} \log \frac{1}{\left|x-y^{*}\right|},
$$

and set

$$
\psi(x, y)=H(x, y)-H^{*}(x, y)
$$

Then from the equation corresponding to $H(x, y)$ and the elliptic regularity theory, it is not difficult to verify $\psi(x, y)$ is bounded in $\bar{\Omega} \times \bar{\Omega}$ and hence one can derive the estimates

$$
H(x, y)=-\frac{1}{2 \pi} \log \frac{1}{\left|x-y^{*}\right|}+O(1) \quad \text { for all } x \in \bar{\Omega} \text { uniformly. }
$$

If $y \in \partial \Omega$, note that $H(x, y)$ satisfies

$$
\begin{cases}\Delta H(x, y)=\frac{1}{|\Omega|} & \text { in } \Omega, \\ \frac{\partial H}{\partial v}(x, y)=\frac{1}{\pi} \frac{(x-y) \cdot v(x)}{|x-y|^{2}} & \text { on } \partial \Omega .\end{cases}
$$

With this and (2-10), we obtain that $x \mapsto H(x, y) \in C^{1, \alpha}(\bar{\Omega})$. On the other hand, by the continuity of the boundary term with respect to $y$ in $L^{\infty}(\partial \Omega)$, we can get $H(x, y) \in C(\bar{\Omega}, \partial \Omega)$. In particular, $H(x, x)$ is in $C(\partial \Omega)$.

Now, we prove the result. It suffices to show the existence of critical points of the function $\varphi_{2+2}\left(\xi_{1}, \ldots, \xi_{4}\right)$ in $\mu_{d}$. In this case,

$$
\begin{array}{r}
\varphi_{2+2}\left(\xi_{1}, \ldots, \xi_{4}\right)=16 \pi^{2}\left(4 H\left(\xi_{1}, \xi_{1}\right)+4 H\left(\xi_{2}, \xi_{2}\right)+H\left(\xi_{3}, \xi_{3}\right)+H\left(\xi_{4}, \xi_{4}\right)\right. \\
-4 G\left(\xi_{1}, \xi_{2}\right)+2 G\left(\xi_{1}, \xi_{3}\right)-2 G\left(\xi_{1}, \xi_{4}\right) \\
\left.-2 G\left(\xi_{2}, \xi_{3}\right)+2 G\left(\xi_{2}, \xi_{4}\right)-G\left(\xi_{3}, \xi_{4}\right)\right) .
\end{array}
$$

We will look for a solution to problem (1-2) with the concentration points $\xi$ given by

$\xi_{1}=(-\lambda, 0), \quad \xi_{2}=(\lambda, 0), \quad \xi_{3}=(1,0), \quad$ and $\quad \xi_{4}=(-1,0) \quad$ for $\lambda \in(0,1)$.

Using results obtained in the previous sections (or from the proof of Theorem 1.2), we reduce the problem of finding solution to (1-2) to that finding critical points of 
the function $\varphi_{2+2}(\lambda):(0,1) \rightarrow \mathbb{R}$ defined by

$$
\begin{aligned}
& \varphi_{2+2}(\lambda):=\varphi_{2+2}(\xi(\lambda)) \\
& =16 \pi^{2}\left(H\left(\xi_{3}, \xi_{3}\right)+H\left(\xi_{4}, \xi_{4}\right)-\frac{4}{\pi} \log \frac{1}{2-\lambda}+O(1)\right. \\
& \quad-\frac{2}{\pi} \log \frac{1}{2 \lambda}-\frac{4}{\pi} \log \frac{1}{1-\lambda}+\frac{4}{\pi} \log \frac{1}{1+\lambda}-\frac{1}{\pi} \log \frac{1}{2} \\
& \left.\quad-H\left(\xi_{1}, \xi_{2}\right)+H\left(\xi_{1}, \xi_{3}\right)-H\left(\xi_{1}, \xi_{4}\right)-H\left(\xi_{2}, \xi_{3}\right)+H\left(\xi_{2}, \xi_{4}\right)-H\left(\xi_{3}, \xi_{4}\right)\right) \\
& =32 \pi(2 \log (2-\lambda)+\log \lambda+2 \log (1-\lambda)-2 \log (1+\lambda))+O(1) .
\end{aligned}
$$

Here, we have used the fact that $H(x, y) \in C(\bar{B}, \partial B)$ and (6-3). Now there exists a $\lambda_{0} \in(0,1)$ such that $\varphi_{2+2}\left(\lambda_{0}\right)=\max _{\lambda \in(0,1)} \varphi_{2+2}(\lambda)$, since $\lim _{\lambda \rightarrow 0^{+}} \varphi_{2+2}(\lambda)=$ $\lim _{\lambda \rightarrow 1^{-}} \varphi_{2+2}(\lambda)=-\infty$. Then $\lambda_{0}$ is a $C^{0}$-stable critical point of $\varphi_{2+2}$, and so the function $\tilde{F}_{\varepsilon}(\xi)$ defined by (6-1) has a critical point. This proves our result.

\section{Appendix A.}

Proof of (2-22) and (2-23). By Lemma 2.1 and the fact that $H$ is $C^{1}$ in $\bar{\Omega}$, we have

$$
\begin{aligned}
H_{j}^{\varepsilon}(\varepsilon y) & =c_{j} H\left(\varepsilon y, \xi_{j}\right)-\log \left(8 \mu_{j}^{2}\right)+O\left(\varepsilon^{\alpha}\right) \\
& =c_{j} H\left(\xi_{i}, \xi_{j}\right)-\log \left(8 \mu_{j}^{2}\right)+O\left(\varepsilon^{\alpha}\right)+O\left(\varepsilon\left|y-\xi_{i}^{\prime}\right|\right) .
\end{aligned}
$$

Let us fix a small constant $\delta>0$. For $\left|y-\xi_{i}^{\prime}\right| \leq \delta / \varepsilon$,

$$
\begin{aligned}
&(-1)^{i-1} H_{i}^{\varepsilon}(\varepsilon y)+\sum_{j \neq i}(-1)^{j-1}\left(\log \frac{8 \mu_{j}^{2}}{\left(\varepsilon^{2} \mu_{i}^{2}+\left|\varepsilon y-\varepsilon \xi_{j}^{\prime}\right|^{2}\right)^{2}}+H_{j}^{\varepsilon}(\varepsilon y)\right) \\
&=(-1)^{i-1}\left(c_{i} H\left(\xi_{i}, \xi_{i}\right)-\log \left(8 \mu_{i}^{2}\right)\right)+O\left(\varepsilon^{\alpha}\right)+O\left(\varepsilon\left|y-\xi_{i}^{\prime}\right|\right) \\
& \quad+\sum_{j \neq i}(-1)^{j-1}\left(\log \frac{8 \mu_{j}^{2}}{\left|\xi_{i}-\xi_{j}\right|^{4}}+c_{j} H\left(\xi_{i}, \xi_{j}\right)-\log \left(8 \mu_{j}^{2}\right)\right) \\
&=(-1)^{i-1}\left(c_{i} H\left(\xi_{i}, \xi_{i}\right)-\log \left(8 \mu_{i}^{2}\right)\right) \quad+O\left(\varepsilon\left|y-\xi_{i}^{\prime}\right|\right) \\
& \quad+\sum_{j \neq i}(-1)^{j-1} c_{j} G\left(\xi_{i}, \xi_{j}\right)+O\left(\varepsilon^{\alpha}\right)+O
\end{aligned}
$$

which is equal to $O\left(\varepsilon^{\alpha}\right)+O\left(\varepsilon\left|y-\xi_{i}^{\prime}\right|\right)$; here first equality follows because

$$
\begin{aligned}
\varepsilon^{2} \mu_{j}^{2}+\varepsilon^{2}\left|y-\xi_{j}^{\prime}\right|^{2} & =\left(\left|\xi_{j}-\xi_{i}\right|+O\left(\left|\varepsilon y-\xi_{i}\right|\right)\right)^{2}+\varepsilon^{2} \mu_{j}^{2} \\
& =\left|\xi_{j}-\xi_{i}\right|^{2}\left(1+O\left(\frac{\left|\varepsilon y-\xi_{i}\right|^{2}}{\left|\xi_{j}-\xi_{i}\right|^{2}}\right)+\frac{\varepsilon^{2} \mu_{j}^{2}}{\left|\xi_{j}-\xi_{i}\right|^{2}}\right) \\
& =\left|\xi_{j}-\xi_{i}\right|^{2}\left(1+O\left(\varepsilon^{2}\left|y-\xi_{i}^{\prime}\right|^{2}\right)+O\left(\varepsilon^{2}\right)\right) .
\end{aligned}
$$


First, we estimate $W$. For $\left|y-\xi_{i}^{\prime}\right| \leq \delta / \varepsilon$, a direct computation shows

$$
\begin{aligned}
& W= 2 \varepsilon^{4} \cosh V \\
&= \varepsilon^{4} \exp \left(\sum_{i=1}^{2(k+l)}(-1)^{i-1}\left(u_{i}+H_{i}^{\varepsilon}\right)\right)+\varepsilon^{4} \exp \left(\sum_{i=1}^{2(k+l)}(-1)^{i}\left(u_{i}+H_{i}^{\varepsilon}\right)\right) \\
&= \varepsilon^{4}\left(\frac{8 \mu_{i}^{2}}{\varepsilon^{4}\left(\mu_{i}^{2}+\left|y-\xi_{i}^{\prime}\right|^{2}\right)^{2}}\right)^{(-1)^{i-1}} \\
& \times \exp \left((-1)^{i-1} H_{i}^{\varepsilon}(\varepsilon y)+\sum_{j \neq i}(-1)^{j-1}\left(\log \frac{8 \mu_{j}^{2}}{\left(\varepsilon^{2} \mu_{j}^{2}+\varepsilon^{2}\left|y-\xi_{j}^{\prime}\right|^{2}\right)^{2}}+H_{j}^{\varepsilon}(\varepsilon y)\right)\right) \\
&+\varepsilon^{4}\left(\frac{8 \mu_{i}^{2}}{\varepsilon^{4}\left(\mu_{i}^{2}+\left|y-\xi_{i}^{\prime}\right|^{2}\right)^{2}}\right)^{(-1)^{i}} \\
& \times \exp \left((-1)^{i} H_{i}^{\varepsilon}(\varepsilon y)+\sum_{j \neq i}(-1)^{j}\left(\log \frac{8 \mu_{j}^{2}}{\left(\varepsilon^{2} \mu_{j}^{2}+\varepsilon^{2}\left|y-\xi_{j}^{\prime}\right|^{2}\right)^{2}}+H_{j}^{\varepsilon}(\varepsilon y)\right)\right) \\
&= \varepsilon^{4}\left(\left(\frac{8 \mu_{i}^{2}}{\varepsilon^{4}\left(\mu_{i}^{2}+\left|y-\xi_{i}^{\prime}\right|^{2}\right)^{2}}\right)^{(-1)^{i-1}}+\left(\frac{8 \mu_{i}^{2}}{\varepsilon^{4}\left(\mu_{i}^{2}+\left|y-\xi_{i}^{\prime}\right|^{2}\right)^{2}}\right)^{(-1)^{i}}\right) \\
&= \frac{8 \mu_{i}^{2}}{\left(\mu_{i}^{2}+\left|y-\xi_{i}^{\prime}\right|^{2}\right)^{2}}\left(1+O\left(\varepsilon^{\alpha}\right)+O\left(\varepsilon\left|y-\xi_{i}^{\prime}\right|\right)\right)+O\left(\varepsilon^{4}\right) . \\
&
\end{aligned}
$$

Therefore

$$
\begin{array}{r}
\text { (A-1) } W(y)=\frac{8 \mu_{i}^{2}}{\left(\mu_{i}^{2}+\left|y-\xi_{i}^{\prime}\right|^{2}\right)^{2}}\left(1+O\left(\varepsilon^{\alpha}\right)+O\left(\varepsilon\left|y-\xi_{i}^{\prime}\right|\right)\right) \\
\text { for all }\left|y-\xi_{i}^{\prime}\right|<\delta / \varepsilon .
\end{array}
$$

Similarly, for $\left|y-\xi_{i}^{\prime}\right|<\delta / \varepsilon$ we have

$2 \varepsilon^{4} \sinh V$

$(\mathrm{A}-2)$

$$
\begin{array}{r}
=\varepsilon^{4}\left(\left(\frac{8 \mu_{i}^{2}}{\varepsilon^{4}\left(\mu_{i}^{2}+\left|y-\xi_{i}^{\prime}\right|^{2}\right)^{2}}\right)^{(-1)^{i-1}}-\right. \\
\left.-\left(\frac{8 \mu_{i}^{2}}{\varepsilon^{4}\left(\mu_{i}^{2}+\left|y-\xi_{i}^{\prime}\right|^{2}\right)^{2}}\right)^{(-1)^{i}}\right) \\
\times \exp \left(O\left(\varepsilon^{\alpha}\right)+O\left(\varepsilon\left|y-\xi_{i}^{\prime}\right|\right)\right) \\
=(-1)^{i-1} \frac{8 \mu_{i}^{2}}{\left(\mu_{i}^{2}+\left|y-\xi_{i}^{\prime}\right|^{2}\right)^{2}}\left(1+O\left(\varepsilon^{\alpha}\right)+O\left(\varepsilon\left|y-\xi_{i}^{\prime}\right|\right)\right)+O\left(\varepsilon^{4}\right) .
\end{array}
$$

On the other hand, for $\left|y-\xi_{i}^{\prime}\right| \geq \delta / \varepsilon$, it is easy to see that $W(y)=O\left(\varepsilon^{4}\right)$ and $2 \varepsilon^{4} \sinh V=O\left(\varepsilon^{4}\right)$. This, together with (A-1), implies (2-23) and (2-24). 
Next, by our definitions,

$$
\begin{aligned}
\Delta V & =\sum_{i=1}^{2(k+l)}(-1)^{i-1}\left(\varepsilon^{2} \Delta u_{i}(\varepsilon y)+\varepsilon^{2} \Delta H_{i}^{\varepsilon}(\varepsilon y)\right) \\
& =\sum_{i=1}^{2(k+l)}(-1)^{i-1}\left(-\varepsilon^{4} e^{u_{i}(\varepsilon y)}+\frac{\varepsilon^{4}}{|\Omega|} \int_{\Omega} e^{u_{i}(x)} d x\right) \\
& =\sum_{i=1}^{2(k+l)}(-1)^{i-1}\left(-\frac{8 \mu_{i}^{2}}{\left(\mu_{i}^{2}+\left|y-\xi_{i}^{\prime}\right|^{2}\right)^{2}}\right)+\sum_{i=1}^{2(k+l)}(-1)^{i-1} \frac{\varepsilon^{4}}{|\Omega|} \int_{\Omega} e^{u_{i}(x)} d x .
\end{aligned}
$$

The last term in the above equality can be controlled by $O\left(\varepsilon^{4}\right)$ since from (2-7), we have

$$
\varepsilon^{2} \sum_{i=1}^{2(k+l)}(-1)^{i-1} \int_{\Omega} e^{u_{i}}=O\left(\varepsilon^{2}\left|\mu_{i}-\mu_{j}\right|\right),
$$

Combining this with (A-2), we get (2-22).

\section{Appendix B.}

Proof of Claim 1. Since $\eta^{\prime}(r)$ has a jump at $r=\varepsilon^{-\gamma}$ and $r=\varepsilon^{-\beta}$ and is otherwise smooth, we see that $L\left(\tilde{Z}_{0 i}\right)$ is a measure.

$$
\begin{aligned}
L\left(\tilde{Z}_{0 i}\right)= & (-\Delta-W)\left(\eta_{1 i} Z_{0 i}+\varepsilon\left(1-\eta_{1 i}\right) \eta_{2 i} \hat{Z}_{0 i}\right) \\
= & -\left(Z_{0 i}-\varepsilon \eta_{2 i} \hat{Z}_{0 i}\right)\left(\left[\eta_{1 i}^{\prime}\left(\varepsilon^{-\gamma}\right)\right] \mu_{\varepsilon^{-\gamma}}+\left[\eta_{1 i}^{\prime}\left(\varepsilon^{-\beta}\right)\right] \mu_{\varepsilon^{-\beta}}\right) \\
& -2 \nabla \eta_{1 i}\left(\nabla Z_{0 i}-\varepsilon \hat{Z}_{0 i} \nabla \eta_{2 i}-\varepsilon \eta_{2 i} \nabla \hat{Z}_{0 i}\right)-\eta_{1 i}\left(\Delta Z_{0 i}+W Z_{0 i}\right) \\
& -\varepsilon\left(1-\eta_{1 i}\right)\left(\hat{Z}_{0 i} \Delta \eta_{2 i}+\eta_{2 i} \Delta \hat{Z}_{0 i}+2 \nabla \eta_{2 i} \nabla \hat{Z}_{0 i}+W \eta_{2 i} \hat{Z}_{0 i}\right)
\end{aligned}
$$

where $\left[\eta_{1 i}^{\prime}(r)\right]=\eta_{1 i}^{\prime}\left(r^{+}\right)-\eta_{1 i}^{\prime}\left(r^{-}\right)$denotes the jump of $\eta_{1 i}^{\prime}$ at $r$, and $\mu_{r}$ is the 1-dimensional measure on the circle of radius $r$.

Let us consider first the case $m=i$ :

$$
\begin{aligned}
\int_{\Omega_{\varepsilon}} \log \left|y_{i}-z\right| L\left(\tilde{Z}_{0 i}\right)=\int_{\Omega_{\varepsilon}}\left(\log \left|y_{i}-z\right|-\log \mid \xi_{i}^{\prime}\right. & -z \mid) L\left(\tilde{Z}_{0 i}\right) d z \\
& +\int_{\Omega_{\varepsilon}} \log \left|\xi_{i}^{\prime}-z\right| L\left(\tilde{Z}_{0 i}\right) d z .
\end{aligned}
$$

Let $r=\left|z-\xi_{i}^{\prime}\right|$, and note that $\Delta \eta_{2 i}=O\left(\varepsilon^{2 \beta}\right)$ and $\nabla \eta_{2 i}=O\left(\varepsilon^{\beta}\right)$. For $r<\varepsilon^{-\beta}$, we have

$$
\begin{aligned}
& \eta_{1 i}\left(\Delta Z_{0 i}+W Z_{0 i}\right)=\eta_{1 i}\left(\Delta Z_{0 i}+e^{v_{i}}\left(1+\theta_{\varepsilon}\right) Z_{0 i}\right) \\
& \quad \leq \frac{8 \mu_{i}^{2}}{\left(\mu_{i}^{2}+\left|z-\xi_{i}^{\prime}\right|^{2}\right)^{2}} O\left(\varepsilon^{\alpha}+\varepsilon\left|z-\xi_{i}^{\prime}\right|\right)+O\left(\frac{\varepsilon^{\alpha}}{\left(1+\left|y-\xi_{i}^{\prime}\right|\right)^{3}}\right) .
\end{aligned}
$$


Thus

(B-3)

$$
\begin{aligned}
& \left|\int_{\Omega_{\varepsilon}} \eta_{1 i}\left(\Delta Z_{0 i}+W Z_{0 i}\right) \log \right| z-\xi_{i}^{\prime}|| \\
& \quad \leq \int_{\Omega_{\varepsilon}} \eta_{1 i}\left(\frac{8 \mu_{i}^{2} O\left(\varepsilon^{\alpha}+\varepsilon\left|z-\xi_{i}^{\prime}\right|\right)}{\left(\mu_{i}^{2}+\left|z-\xi_{i}^{\prime}\right|^{2}\right)^{2}}+O\left(\frac{\varepsilon^{\alpha}}{\left(1+\left|y-\xi_{i}^{\prime}\right|\right)^{3}}\right)\right) \log \left|z-\xi_{i}^{\prime}\right| \\
& \quad \leq C \int_{0}^{\varepsilon^{-\beta}}\left(\frac{\varepsilon^{\alpha}}{(1+r)^{3}}+\frac{\varepsilon^{\alpha}+\varepsilon r}{\left(1+r^{2}\right)^{2}}\right) r \log r d r \\
& \quad=O\left(\left(\varepsilon^{\alpha}+\varepsilon^{1-\beta}\right) \log \varepsilon^{-1}\right) \\
& \quad=o(1) .
\end{aligned}
$$

For $\varepsilon^{-\gamma}<r<\varepsilon^{-\beta}$,

$$
\begin{aligned}
\frac{1}{\mu_{i}}-a_{0 i} G\left(\varepsilon z, \xi_{i}\right) & =\frac{1}{\mu_{i}}-\frac{4 \log \varepsilon^{-1}-4 \log \left|z-\xi_{i}^{\prime}\right|+c_{i} H\left(\varepsilon z, \xi_{i}\right)}{\mu_{i}\left[4(1-\gamma) \log \varepsilon^{-1}+c_{i} H\left(\xi_{i}, \xi_{j}\right)\right]} \\
& =\frac{\log r-\gamma \log \varepsilon^{-1}+\varepsilon r}{(1-\gamma) \mu_{i} \log \varepsilon^{-1}}(1+O(\varepsilon)) .
\end{aligned}
$$

Therefore,

$$
\begin{aligned}
\int_{\Omega_{\varepsilon}}(1 & \left.-\eta_{1 i}\right) W\left(\mu_{i}^{-1}-a_{0 i} G\right) \log \left|z-\xi_{i}^{\prime}\right| d z \\
& =\int_{r>\varepsilon^{-\gamma}} O\left(\frac{\log r-\gamma \log \varepsilon^{-1}+\varepsilon r}{(1-\gamma) \mu_{i} \log \varepsilon^{-1}}\right) O\left(r^{-4 r}\right) \log r d r \\
& =O\left(\varepsilon^{2 \gamma} \log \varepsilon^{-1}\right)
\end{aligned}
$$

and

(B-6)

$$
\begin{aligned}
\int_{\Omega_{\varepsilon}} \nabla & \eta_{1 i}\left(\nabla Z_{0 i}-\varepsilon \hat{Z}_{0 i} \nabla \eta_{2 i}-\varepsilon \eta_{2 i} \nabla \hat{Z}_{0 i}\right) \log \left|z-\xi_{i}^{\prime}\right| d z \\
=2 \pi \int_{\varepsilon^{-\gamma}}^{\varepsilon^{-\beta}} & \frac{-r^{-1}}{(\beta-\gamma) \log \varepsilon^{-1}} \\
& \times\left(O\left(r^{-3}\right)+O\left(\varepsilon^{1+\beta}\right)+O\left(\frac{\varepsilon}{\log \varepsilon^{-1}}\left(r^{-1}+C\right)\right)\right) r \log r d r \\
=O\left(\varepsilon^{2 \gamma}\right) & +O\left(\frac{\varepsilon^{1-\beta}}{\log \varepsilon^{-1}}\right) .
\end{aligned}
$$

For $r>\varepsilon^{-\gamma}$,

$$
\begin{aligned}
& \hat{Z}_{0 i} \Delta \eta_{2 i}+\eta_{2 i} \Delta \hat{Z}_{0 i}+2 \nabla \eta_{2 i} \nabla \hat{Z}_{0 i}+W \eta_{2 i} \hat{Z}_{0 i} \\
& \quad=\hat{Z}_{0 i} \Delta \eta_{2 i}+2 \nabla \eta_{2 i} \nabla \hat{Z}_{0 i}+\eta_{2 i}\left(\Delta Z_{0 i}+W Z_{0 i}+a_{0 i} \Delta G-W \mu_{i}^{-1}+W a_{2 i} G\right) .
\end{aligned}
$$


So, recalling (B-5), we have

$$
\begin{aligned}
& \varepsilon \int_{\Omega_{\varepsilon}}\left(1-\eta_{1 i}\right)\left(\hat{Z}_{0 i} \Delta \eta_{2 i}+\eta_{2 i} \Delta \hat{Z}_{0 i}+2 \nabla \eta_{2 i} \nabla \hat{Z}_{0 i}+W \eta_{2 i} \hat{Z}_{0 i}\right) \log \left|z-\xi_{i}^{\prime}\right| d z \\
& =\varepsilon \int_{\varepsilon^{-\beta}}^{2 \varepsilon^{-\beta}} O\left(\varepsilon^{2 \beta}\right) r \log r d r+\varepsilon \int_{\varepsilon^{-\beta}}^{2 \varepsilon^{-\beta}} O\left(\varepsilon^{\beta}\right) O\left(r^{-3}+\frac{\varepsilon}{\log \varepsilon^{-1}}\left(C+r^{-1}\right)\right) r \log r d r \\
& \quad+\varepsilon \int_{\varepsilon^{-\gamma}}^{2 \varepsilon^{-\beta}}\left(O\left(\frac{\varepsilon^{\alpha}+\varepsilon r}{r^{4}}\right)+O\left(\frac{\varepsilon^{\alpha}}{(1+r)^{3}}\right)+O\left(\frac{\varepsilon^{2}}{\log \varepsilon^{-1}}\right)\right) r \log r d r \\
& \quad-\varepsilon \int_{\Omega_{\varepsilon}}\left(1-\eta_{1 i}\right) W\left(\mu_{i}^{-1}-a_{0 i} G\right) \log \left|z-\xi_{i}^{\prime}\right| d z,
\end{aligned}
$$

which is equal to $O\left(\varepsilon \log \varepsilon^{-1}\right)$. A direct computation shows

$$
\begin{aligned}
\int_{\Omega_{\varepsilon}}\left[\eta_{1 i}^{\prime}\left(\varepsilon^{-\gamma}\right)\right] & \mu_{\varepsilon^{-\gamma}}\left(Z_{0 i}-\varepsilon \eta_{2 i} \hat{Z}_{0 i}\right) \log \left|z-\xi_{i}^{\prime}\right| d z \\
& =\frac{-\varepsilon^{\gamma}}{(\beta-\gamma) \log \varepsilon^{-1}} \int_{r=\varepsilon^{-\gamma}}\left(Z_{0 i}-\varepsilon \hat{Z}_{0 i}\right) \log \left|z-\xi_{i}^{\prime}\right| \\
& =\frac{-\varepsilon^{\gamma}}{(\beta-\gamma) \log \varepsilon^{-1}} \times \frac{1+O\left(\varepsilon^{2 \gamma}\right)}{\mu_{i}} \times 2 \pi \varepsilon^{-\gamma} \log \varepsilon^{-\gamma} \\
& =\frac{-2 \pi \gamma}{\mu_{i}(\beta-\gamma)}+O\left(\varepsilon^{2 \gamma}\right) .
\end{aligned}
$$

Similarly,

$$
\int_{\Omega_{\varepsilon}}\left[\eta_{1 i}^{\prime}\left(\varepsilon^{-\beta}\right)\right] \mu_{\varepsilon^{-\beta}}\left(Z_{0 i}-\varepsilon \eta_{2 i} \hat{Z}_{0 i}\right) \log \left|z-\xi_{i}^{\prime}\right| d z=\frac{2 \pi \beta}{\mu_{i}(\beta-\gamma)}+O\left(\varepsilon^{2 \beta}\right) .
$$

Hence

$$
\int_{\Omega_{\varepsilon}} L\left(\tilde{Z}_{0 i}\right) \log \left|z-\xi_{i}^{\prime}\right| d z=\frac{2 \pi}{\mu_{i}}+o(1) .
$$

For the first integral in the right side of (B-1), we can assume $R_{\varepsilon} \rightarrow+\infty$ slowly enough so that $\varepsilon^{\gamma} R_{\varepsilon} \rightarrow 0$. Then

$$
|\log | y_{i}-z|-\log | \xi_{i}^{\prime}-z||=\left|\log \frac{\left|y_{i}-z\right|}{r}\right| \leq\left|\log \frac{\left|y_{i}-\xi_{i}^{\prime}\right|+r}{r}\right|
$$

for $r=\left|\xi_{i}^{\prime}-z\right|$; therefore we have from (B-2)

$$
\left|\int_{\Omega_{\varepsilon}}\left(\log \left|y_{i}-z\right|-\log \left|\xi_{i}^{\prime}-z\right|\right) \eta_{1 i}\left(\Delta Z_{0 i}+W Z_{0 i}\right) d z\right|
$$

$$
\begin{aligned}
& \leq C \int_{0}^{\varepsilon^{-\beta}} \log \left(R_{\varepsilon} r^{-1}+1\right)\left(O\left(\frac{\varepsilon^{\alpha}+\varepsilon r}{\left(1+r^{2}\right)^{2}}\right)+O\left(\frac{\varepsilon^{\alpha}}{(1+r)^{3}}\right)\right) r d r \\
& =O\left(\varepsilon^{\alpha}\left(R_{\varepsilon}+\log \varepsilon^{-1}\right)\right) .
\end{aligned}
$$


On the other hand, from (B-7), for $\varepsilon^{-\gamma} \leq r=\left|z-\xi_{i}^{\prime}\right| \leq \varepsilon^{-\beta}$ we have

$$
|\log | y_{i}-z|-\log | \xi_{i}^{\prime}-z|| \leq C\left|y_{i}-\xi_{i}^{\prime}\right| / \varepsilon^{-\gamma}
$$

and it follows that

$$
\left|\int_{\Omega_{\varepsilon}}\left(\log \left|y_{i}-z\right|-\log \left|\xi_{i}^{\prime}-z\right|\right)\left(L\left(\tilde{Z}_{0 i}\right)+\eta_{1 i}\left(\Delta Z_{0 i}+W Z_{0 i}\right)\right) d z\right|=O\left(\varepsilon^{\gamma} R_{\varepsilon}\right) .
$$

Thus, from this and (B-8), we obtain

$$
\left|\int_{\Omega_{\varepsilon}}\left(\log \left|y_{i}-z\right|-\log \left|\xi_{i}^{\prime}-z\right|\right) L\left(\tilde{Z}_{0 i}\right)\right|=o(1) .
$$

Next, we show that if $m \neq i$, then

$$
\int_{\Omega_{\varepsilon}} \log \left|y_{m}-z\right| L\left(\tilde{Z}_{0 i}\right) d z=o(1) .
$$

In fact,

$$
\begin{aligned}
& \int_{\Omega_{\varepsilon}} \log \left|y_{m}-z\right| L\left(\tilde{Z}_{0 i}\right) d z \\
& \quad=\int_{\Omega_{\varepsilon}}\left(\log \left|y_{m}-z\right|-\log \left|y_{m}-\xi_{i}^{\prime}\right|\right) L\left(\tilde{Z}_{0 i}\right) d z+\int_{\Omega_{\varepsilon}} \log \left|y_{m}-\xi_{i}^{\prime}\right| L\left(\tilde{Z}_{0 i}\right) d z .
\end{aligned}
$$

We assume that $R_{\varepsilon}<\varepsilon^{-\gamma} / 2$, so that

$$
|\log | y_{m}-z|-\log | y_{m}-\xi_{i}^{\prime}|| \leq \log \left(1+\frac{\left|z-\xi_{i}^{\prime}\right|}{\left|y_{m}-\xi_{i}^{\prime}\right|}\right)=O\left(\varepsilon\left|z-\xi_{i}^{\prime}\right|\right) .
$$

Thus

$$
\left|\int_{\Omega_{\varepsilon}}\left(\log \left|y_{m}-z\right|-\log \left|y_{m}-\xi_{i}^{\prime}\right|\right) L\left(\tilde{Z}_{0 i}\right) d z\right|=O\left(\frac{\varepsilon^{1-\beta}}{\log \varepsilon^{-1}}\right) .
$$

Finally,

$$
\int_{\Omega_{\varepsilon}} L\left(\tilde{Z}_{0 i}\right) d z=O\left(\varepsilon^{2 \gamma}\right)
$$

This implies

$$
\int_{\Omega_{\varepsilon}} \log \left|y_{m}-\xi_{i}^{\prime}\right| L\left(\tilde{Z}_{0 i}\right) d z=o(1) .
$$

Therefore Claim 1 holds.

Proof of Claim 4. Let

$$
\zeta(r)= \begin{cases}1 & \text { if } r<\varepsilon^{-1 / 2}, \\ (\log (\delta / \varepsilon)-\log r) /\left(\log (\delta / \varepsilon)-\log \varepsilon^{-1 / 2}\right) & \text { if } \varepsilon^{-1 / 2}<r<\delta / \varepsilon, \\ 0 & \text { if } r>\delta / \varepsilon\end{cases}
$$


and set

$$
\psi(z)=\sum_{i=1}^{2(k+l)} H\left(\varepsilon y, \xi_{i}\right) \zeta\left(\left|z-\xi_{i}^{\prime}\right|\right)
$$

Testing (3-9) by $\psi$ and integrating by parts, we obtain

$$
\int_{\Omega_{\varepsilon}}\left(W \tilde{\phi}+h-\sum_{i=1}^{2(k+l)} d_{i} L\left(\tilde{Z}_{0 i}\right)\right) \psi+\int_{\Omega_{\varepsilon}} \tilde{\phi} \Delta \psi-\int_{\partial \Omega_{\varepsilon}} \tilde{\phi} \frac{\partial \psi}{\partial v}=0 .
$$

Thus

$$
A=\int_{\Omega_{\varepsilon}}(H(\varepsilon y, \varepsilon z)-\psi)\left(W \tilde{\phi}+h-\sum_{i=1}^{2(k+l)} d_{i} L\left(\tilde{Z}_{0 i}\right)\right)-\int_{\Omega_{\varepsilon}} \tilde{\phi} \Delta \psi+\int_{\partial \Omega_{\varepsilon}} \tilde{\phi} \frac{\partial \psi}{\partial \nu} .
$$

Since $H, \psi$ and $\tilde{\phi}$ are bounded,

$$
\left|\int_{\Omega_{\varepsilon}}(H(\varepsilon y, \varepsilon z)-\psi) h d z\right| \leq C\|h\|_{*}=o(1)
$$

and

$$
\left|\int_{\Omega_{\varepsilon}}(H(\varepsilon y, \varepsilon z)-\psi) L\left(\tilde{Z}_{0 i}\right)\right| \leq C\left|\int_{\Omega_{\varepsilon}} L\left(\tilde{Z}_{0 i}\right) d z\right|=o(1) .
$$

Also, it is not difficult to show that

(B-13) $\int_{\Omega_{\varepsilon}} \tilde{\phi} \Delta \psi=O\left(\frac{1}{\log (\delta / \varepsilon)}\right)=o(1), \quad \int_{\partial \Omega_{\varepsilon}} \tilde{\phi} \frac{\partial \psi}{\partial v}=O\left(\frac{1}{\log (\delta / \varepsilon)}\right)=o(1)$.

For instance, the first integer in (B-13) can be estimated as

$$
\left|\int_{\Omega_{\varepsilon}} \tilde{\phi} \Delta \psi\right| \leq\|\tilde{\phi}\|_{L^{\infty}\left(\Omega_{\varepsilon}\right)} \int_{\Omega_{\varepsilon}}|\Delta \psi|
$$

But $\Delta \psi$ is a measure with support on the arcs $r=\varepsilon^{-1 / 2}$ and $r=\delta / \varepsilon$, where $r=\left|z-\xi_{i}^{\prime}\right|$, and

$$
\int_{\Omega_{\varepsilon}}|\Delta \psi|=O\left(\varepsilon^{-1 / 2} \frac{1}{\varepsilon^{-1 / 2} \log \varepsilon^{-1}}+\frac{\delta}{\varepsilon} \frac{1}{(\delta / \varepsilon) \log \varepsilon^{-1}}\right)=O\left(\frac{1}{\log (\delta / \varepsilon)}\right)=o(1) .
$$

Note that for $\left|z-\xi_{i}^{\prime}\right|>\delta / \varepsilon$, we have $W=O\left(r^{-4}\right)$, and $H$ and $\tilde{\phi}$ are bounded; thus

$$
\int_{\Omega_{\varepsilon} \backslash\left(\cup_{i} B_{\delta / \varepsilon}\left(\xi_{i}^{\prime}\right)\right)}(H(\varepsilon y, \varepsilon z)-\psi) W \tilde{\phi}=o(1) .
$$


On the other hand, for $\left|z-\xi_{i}^{\prime}\right| \leq \delta / \varepsilon$, we have $H(\varepsilon y, \varepsilon z)-H\left(\varepsilon y, \xi_{i}\right)=O\left(\varepsilon\left|z-\xi_{i}^{\prime}\right|\right)$ and $W=O\left(\left(r^{2}+1\right)^{-2}\right)$. So

(B-15)

$$
\begin{aligned}
& \left|\int_{\Omega_{\varepsilon} \cap B_{\varepsilon^{-1 / 2}}\left(\xi_{i}^{\prime}\right)}(H(\varepsilon y, \varepsilon z)-\psi(z)) W \tilde{\phi} d z\right| \\
& \quad=\left|\int_{\Omega_{\varepsilon} \cap B_{\varepsilon^{-1 / 2}\left(\xi_{i}^{\prime}\right)}}\left(H(\varepsilon y, \varepsilon z)-H\left(\varepsilon y, \xi_{i}\right)\right) W \tilde{\phi} d z\right| \\
& \leq C \varepsilon \int_{0}^{\varepsilon^{-1 / 2}} \frac{r^{2}}{\left(r^{2}+1\right)^{2}} d r=O\left(\varepsilon^{1 / 2}\right)=o(1) .
\end{aligned}
$$

In the region $\varepsilon^{-1 / 2}<r=\left|z-\xi_{i}^{\prime}\right|<\delta / \varepsilon$, noting the fact that $H, \zeta$ and $\tilde{\phi}$ are bounded and that $W=O\left(r^{-4}\right)$, we find

(B-16) $\left|\int_{\Omega_{\varepsilon} \cap B_{\delta / \varepsilon}\left(\xi_{i}^{\prime}\right) \backslash B_{1 / \sqrt{\varepsilon}}\left(\xi_{i}^{\prime}\right)}(H(\varepsilon y, \varepsilon z)-\psi(z)) W \tilde{\phi} d z\right| \leq C \int_{1 / \sqrt{\varepsilon}}^{\delta / \varepsilon} r^{-3} d r=o(1)$.

Therefore, Claim 4 follows from (B-10)-(B-16).

\section{References}

[Bartolucci and Pistoia 2007] D. Bartolucci and A. Pistoia, "Existence and qualitative properties of concentrating solutions for the sinh-Poisson equation", IMA J. Appl. Math. 72:6 (2007), 706-729. MR 2008k:35130 Zbl 1154.35072

[Brezis and Coron 1984] H. Brezis and J.-M. Coron, "Multiple solutions of $H$-systems and Rellich's conjecture", Comm. Pure Appl. Math. 37:2 (1984), 149-187. MR 85i:53010

[Chen and Lin 2002] C.-C. Chen and C.-S. Lin, "Sharp estimates for solutions of multi-bubbles in compact Riemann surfaces", Comm. Pure Appl. Math. 55:6 (2002), 728-771. MR 2003d:53056 Zbl 1040.53046

[Chow et al. 1998] K. W. Chow, N. W. M. Ko, R. C. K. Leung, and S. K. Tang, "Inviscid twodimensional vortex dynamics and a soliton expansion of the sinh-Poisson equation", Phys. Fluids 10:5 (1998), 1111-1119. MR 99a:76020 Zbl 1185.35184

[Dávila et al. 2005] J. Dávila, M. del Pino, and M. Musso, "Concentrating solutions in a twodimensional elliptic problem with exponential Neumann data”, J. Funct. Anal. 227:2 (2005), 430490. MR 2006g:35083 Zbl 02231228

[Esposito et al. 2006] P. Esposito, A. Pistoia, and J. Wei, "Concentrating solutions for the Hénon equation in $\mathbb{R}^{2}$ ", J. Anal. Math. 100 (2006), 249-280. MR 2009b:35106 Zbl 1173.35504

[Gurarie and Chow 2004] D. Gurarie and K. W. Chow, "Vortex arrays for sinh-Poisson equation of two-dimensional fluids: equilibria and stability", Phys. Fluids 16:9 (2004), 3296-3305. MR 2005b: 76026 Zbl 1187.76196

[Jost et al. 2008] J. Jost, G. Wang, D. Ye, and C. Zhou, "The blow up analysis of solutions of the elliptic sinh-Gordon equation”, Calc. Var. Partial Differential Equations 31:2 (2008), 263-276. MR 2009h:35131 Zbl 1137.35061

[Kuvshinov and Schep 2000] B. N. Kuvshinov and T. J. Schep, "Double-periodic arrays of vortices", Phys. Fluids 12:12 (2000), 3282-3284. MR 2001k:76020 Zbl 1184.76305

[Mallier and Maslowe 1993] R. Mallier and S. A. Maslowe, "A row of counter-rotating vortices", Phys. Fluids A 5:4 (1993), 1074-1075. MR 94a:76018 Zbl 0778.76022 
[Ohtsuka and Suzuki 2006] H. Ohtsuka and T. Suzuki, "Mean field equation for the equilibrium turbulence and a related functional inequality", Adv. Differential Equations 11:3 (2006), 281-304. MR 2007a:53082 Zbl 1109.26014

[del Pino and Wei 2006] M. del Pino and J. Wei, "Collapsing steady states of the Keller-Segel system”, Nonlinearity 19:3 (2006), 661-684. MR 2007b:35130 Zbl 1137.35007

[del Pino et al. 2005] M. del Pino, M. Kowalczyk, and M. Musso, "Singular limits in Liouvilletype equations", Calc. Var. Partial Differential Equations 24:1 (2005), 47-81. MR 2006h:35089 Zbl 1088.35067

[Probstein 1994] R. F. Probstein, Physicochemical Hydrodynamics: An Introduction, Wiley, New York, 1994.

[Spruck 1988] J. Spruck, "The elliptic sinh Gordon equation and the construction of toroidal soap bubbles", pp. 275-301 in Calculus of variations and partial differential equations (Trento, 1986), edited by S. Hildebrandt et al., Lecture Notes in Math. 1340, Springer, Berlin, 1988. MR 90i:35265 Zbl 0697.35044

[Steffen 1986] K. Steffen, "On the nonuniqueness of surfaces with constant mean curvature spanning a given contour”, Arch. Rational Mech. Anal. 94:2 (1986), 101-122. MR 87i:53012 Zbl 0678.49036

[Struwe 1986] M. Struwe, "Nonuniqueness in the Plateau problem for surfaces of constant mean curvature", Arch. Rational Mech. Anal. 93:2 (1986), 135-157. MR 87c:53014 Zbl 0603.49027

[Wei 2009] L. Wei, "On the number of nodal bubbling solutions to a sinh-Poisson equation", Houston J. Math. 35:1 (2009), 291-326. MR 2010b:35141 Zbl 1171.35054

[Wei et al. 2011] J. C. Wei, L. Wei, and F. Zhou, "Concentrating solutions for some Neumann problem with equilibrium vortices", preprint, 2011.

[Wente 1986] H. C. Wente, "Counterexample to a conjecture of H. Hopf", Pacific J. Math. 121:1 (1986), 193-243. MR 87d:53013 Zbl 0586.53003

Received January 3, 2010.

JUNCHENG WEI

DEPARTMENT OF MATHEMATICS

THE Chinese University OF HONG Kong

ROOM 220, LADY SHAW BUILDING

SHATIN, HONG KONG

HONG KONG

wei@math.cuhk.edu.hk

LONG WEI

Institute of APPlied Mathematics AND ENGINEERING COMPUTATions

HANGZHOU DIANZI UNIVERSITY

HANGZHOU, ZHEJIANG 310018

CHINA

alongwei@gmail.com

FENG ZHOU

DEPARTMENT OF MATHEMATICS

EAST CHINA NORMAL UNIVERSITY

SHANGHAI 200062

CHINA

fzhou@math.ecnu.edu.cn 


\title{
PACIFIC JOURNAL OF MATHEMATICS
}

\author{
http://www.pjmath.org \\ Founded in 1951 by
}

E. F. Beckenbach (1906-1982) and F. Wolf (1904-1989)

\section{EDITORS}

V. S. Varadarajan (Managing Editor)

Department of Mathematics

University of California

Los Angeles, CA 90095-1555

pacific@math.ucla.edu

Vyjayanthi Chari

Department of Mathematics

University of California

Riverside, CA 92521-0135

chari@math.ucr.edu

\section{Robert Finn}

Department of Mathematics Stanford University

Stanford, CA 94305-2125

finn@math.stanford.edu

Kefeng Liu

Department of Mathematics

University of California

Los Angeles, CA 90095-1555

liu@math.ucla.edu
Darren Long

Department of Mathematics

University of California

Santa Barbara, CA 93106-3080

long@math.ucsb.edu

Jiang-Hua Lu

Department of Mathematics

The University of Hong Kong

Pokfulam Rd., Hong Kong jhlu@maths.hku.hk

Alexander Merkurjev

Department of Mathematics University of California

Los Angeles, CA 90095-1555 merkurev@math.ucla.edu
Sorin Popa

Department of Mathematics

University of California

Los Angeles, CA 90095-1555

popa@math.ucla.edu

Jie Qing

Department of Mathematics

University of California

Santa Cruz, CA 95064

qing@ cats.ucsc.edu

Jonathan Rogawski

Department of Mathematics

University of California

Los Angeles, CA 90095-1555

jonr@math.ucla.edu

\section{PRODUCTION}

pacific@math.berkeley.edu

Silvio Levy, Scientific Editor Matthew Cargo, Senior Production Editor

ACADEMIA SINICA, TAIPEI

CALIFORNIA INST. OF TECHNOLOGY

INST. DE MATEMÁTICA PURA E APLICADA

KEIO UNIVERSITY

MATH. SCIENCES RESEARCH INSTITUTE

NEW MEXICO STATE UNIV.

OREGON STATE UNIV.

\section{SUPPORTING INSTITUTIONS}

STANFORD UNIVERSITY
UNIV. OF BRITISH COLUMBIA
UNIV. OF CALIFORNIA, BERKELEY
UNIV. OF CALIFORNIA, DAVIS
UNIV. OF CALIFORNIA, LOS ANGELES
UNIV. OF CALIFORNIA, RIVERSIDE
UNIV. OF CALIFORNIA, SAN DIEGO
UNIV. OF CALIF., SANTA BARBARA

UNIV. OF CALIF., SANTA CRUZ

UNIV. OF MONTANA

UNIV. OF OREGON

UNIV. OF SOUTHERN CALIFORNIA

UNIV. OF UTAH

UNIV. OF WASHINGTON

WASHINGTON STATE UNIVERSITY

These supporting institutions contribute to the cost of publication of this Journal, but they are not owners or publishers and have no responsibility for its contents or policies.

See inside back cover or www.pjmath.org for submission instructions.

The subscription price for 2011 is US \$420/year for the electronic version, and \$485/year for print and electronic.

Subscriptions, requests for back issues from the last three years and changes of subscribers address should be sent to Pacific Journal of Mathematics, P.O. Box 4163, Berkeley, CA 94704-0163, U.S.A. Prior back issues are obtainable from Periodicals Service Company, 11 Main Street, Germantown, NY 12526-5635. The Pacific Journal of Mathematics is indexed by Mathematical Reviews, Zentralblatt MATH, PASCAL CNRS Index, Referativnyi Zhurnal, Current Mathematical Publications and the Science Citation Index.

The Pacific Journal of Mathematics (ISSN 0030-8730) at the University of California, c/o Department of Mathematics, 969 Evans Hall, Berkeley, CA 94720-3840, is published monthly except July and August. Periodical rate postage paid at Berkeley, CA 94704, and additional mailing offices. POSTMASTER: send address changes to Pacific Journal of Mathematics, P.O. Box 4163, Berkeley, CA 94704-0163.

PJM peer review and production are managed by EditFLOW ${ }^{\mathrm{TM}}$ from Mathematical Sciences Publishers.

PUBLISHED BY PACIFIC JOURNAL OF MATHEMATICS

at the University of California, Berkeley 94720-3840

A NON-PROFIT CORPORATION

Typeset in IATEX

Copyright $(2011$ by Pacific Journal of Mathematics 


\section{PACIFIC JOURNAL OF MATHEMATICS}

Volume $250 \quad$ No. $1 \quad$ March 2011

Nonconventional ergodic averages and multiple recurrence for von $\quad 1$ Neumann dynamical systems

Tim Austin, TAnja EIsner and Terence TaO

Principal curvatures of fibers and Heegaard surfaces

WILLIAM BRESLIN

Self-improving properties of inequalities of Poincaré type on $s$-John domains

SENG-KeE CHUA and RICHARD L. WHEEDEN

The orbit structure of the Gelfand-Zeitlin group on $n \times n$ matrices

MARK COLARUSSO

On Maslov class rigidity for coisotropic submanifolds

VIKTOR L. GINZBURG

Dirac cohomology of Wallach representations

Jing-Song HuAng, PAVle PANDŽIĆ and Victor PROTSAK An example of a singular metric arising from the blow-up limit in the 191 continuity approach to Kähler-Einstein metrics

YALONG SHI and XIAOHUA ZHU

Detecting when a nonsingular flow is transverse to a foliation

SANDRA SHIELDS

Mixed interior and boundary nodal bubbling solutions for a sinh-Poisson equation

JUNCHENG WeI, LONG WEI and FENG ZHOU 